\title{
BOC Signal Acquisition Algorithm Based on Similar Enfoldment
}

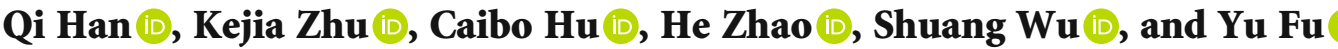 \\ Beijing Satellite Navigation Center, China \\ Correspondence should be addressed to Qi Han; herberthan@foxmail.com, Shuang Wu; wushuang10@tsinghua.org.cn, \\ and Yu Fu; wisherfy@163.com
}

Received 20 August 2019; Revised 28 October 2019; Accepted 4 December 2019; Published 25 February 2020

Academic Editor: Paolo Castaldi

Copyright @ 2020 Qi Han et al. This is an open access article distributed under the Creative Commons Attribution License, which permits unrestricted use, distribution, and reproduction in any medium, provided the original work is properly cited.

\begin{abstract}
A BOC signal has been widely used in the Global Navigation Satellite System (GNSS). Although the BOC signal has many advantages, the autocorrelation function (ACF) of the BOC signal has lots of peaks, which makes signal acquisition difficult. This paper proposes a similar enfoldment acquisition (SEA) algorithm, which can be applied to sin-BOC and cos-BOC signals, with even and odd modulation orders. The SEA algorithm utilizes the similarity between the main peak and the secondary peak to construct a new ACF to eliminate the ambiguity of the BOC signal. This paper simulates the acquisition performance of the SEA algorithm, Martin algorithm, and SCPC algorithm, while the simulation result shows that the SEA algorithm is superior to the Martin algorithm and SCPC algorithm. Since the SEA algorithm does not need filters or auxiliary signals, its complexity is lower than that of the Martin algorithm and SCPC algorithm.
\end{abstract}

\section{Introduction}

In recent years, the Global Navigation Satellite System (GNSS) has developed rapidly. Major countries or regions have established their own satellite navigation systems, such as the Global Positioning System (GPS) of the United States, the Galileo Satellite Navigation System of Europe (Galileo), the BeiDou Navigation Satellite System (BDS) of China, and the Global Navigation Satellite System (GLONASS) of Russia. Since the frequency bands of the major navigation systems are close to each other and the modulated signals become more and more, in order to save the frequency band resources, a binary offset carrier (BOC) signal appears [1].

A BOC $(m, n)$ signal is obtained by Pseudorandom Noise (PRN) codes multiplied with rectangular subcarriers, where the PRN code rate is $f_{n}=n * f_{0}$, the subcarrier frequency is $f_{m}=m * f_{0}$, the reference frequency is $f_{0}=1.023 \mathrm{MHz}$, and the modulation order of the BOC signal is $M=2 \mathrm{~m} / \mathrm{n}$. According to whether the subcarrier phase is sinusoidal or cosine, the BOC signal can be divided into sin-BOC $(m, n)$ and cos-BOC $(m, n)$.

The BOC signal has many advantages: Firstly, owing to the spectral splitting characteristic of the BOC signal [2], BOC signal does not interfere with the original BPSK signal.
Secondly, compared with the BPSK signal, the autocorrelation peak of the BOC signal is narrower, which leads to the BOC signal with higher tracking accuracy and better antimultipath performance [3]. However, due to the multipeak nature of BOC signal ACF, ambiguity is prone to occur in the phase of signal acquisition and tracking.

In order to eliminate the ambiguity of BOC signal ACF, plenty of methods have been proposed by scholars all over the world. The main ideas are as follows: capturing the BOC signal with the acquisition method similar to the BPSK signal $[2,4-8]$, constructing auxiliary signals to eliminate the ambiguity of BOC signal ACF [3, 9-16], and constructing a filter to eliminate ambiguity of ACF [17]. Literature [5, 6] (hereinafter referred to as the Fishman method) filters the upper (lower) sideband of the received signal and the local BOC code, respectively, and after that processes the upper (lower) sideband separately. The authors of $[7,8]$ (hereinafter referred to as the Martin method) uses a filter, whose bandwidth includes the two main lobes of the received signal and all the secondary lobes between the main ones, to process the received signal. Generally speaking, a high complexity turns out due to applying filters to filter out the desired signals in References [5-8]. As demonstrated in [9], an autocorrelation side-peak cancellation technique (ASPeCT) 


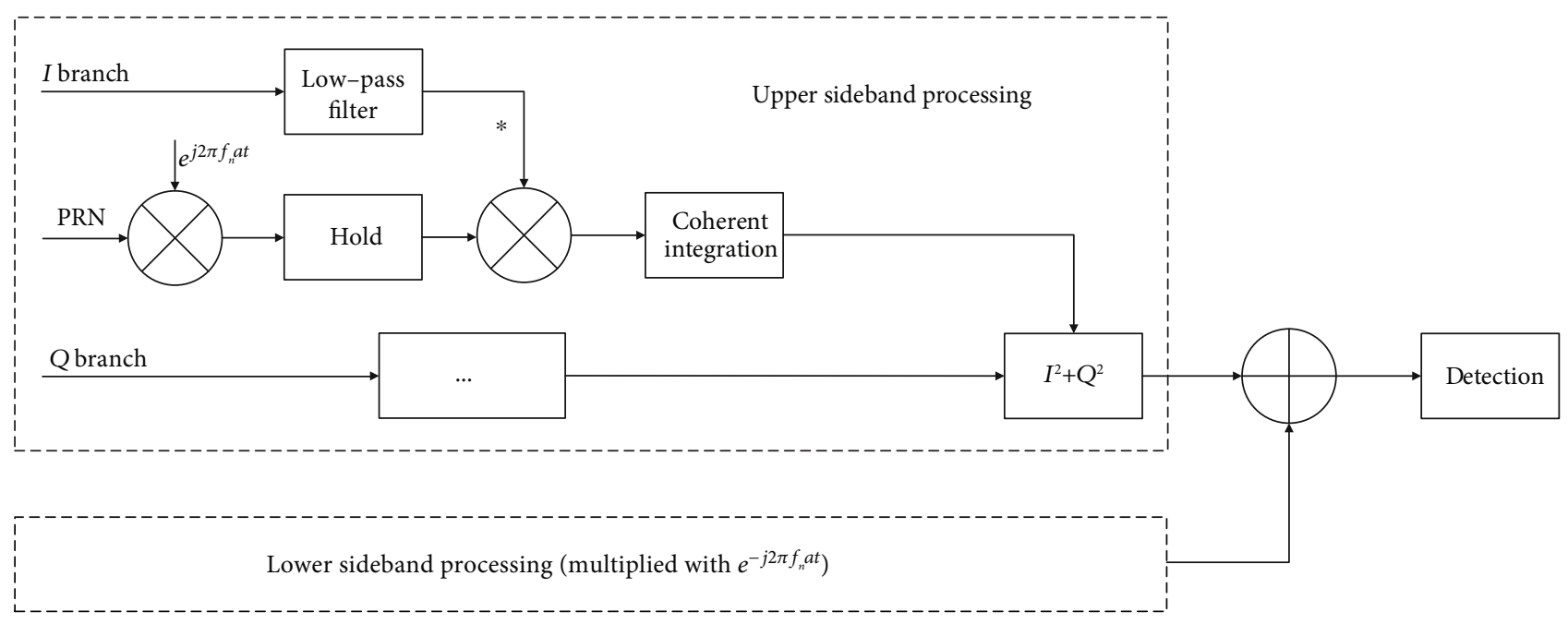

Figure 1: Flow chart of the Martin method.

algorithm is proposed to eliminate redundant edge peaks through a local auxiliary BPSK signal, whereas the ASPeCT method is only applicable to sin-BOC $(n, n)$ signals. A general removing ambiguity via side-peak suppression (GRASS), which uses a local auxiliary signal and is suitable for sin-BOC $(k n, n)$ signals, is proposed in [3]. The GRASS algorithm is improved by two auxiliary signals to make the algorithm suitable for cos-BOC $(m, n)$ signals in literature [10]. Subcarrier phase cancellation (SCPC) technology $[8,11,12]$ eliminates ambiguity of ACF by two auxiliary signals. In Reference [13], the ambiguity of ACF is eliminated by two symmetrical auxiliary signals, which is applicable to all $\operatorname{BOC}(m, n)$ signals. Other methods of constructing auxiliary signals can be referred to References [14-16]. In literature [17], a filter is constructed to process the ACF, and an unambiguous ACF is finally obtained. The acquisition for the GPS L1C signal, which is also an application of the BOC signal, is studied in [18]. A 2-D compressed correlator technique for the fast acquisition of BOC $(m, n)$ signals is proposed in Reference [19]. Yang reconstructs $[20,21]$ the BOC signal ACF by the removing zeros of crosscorrelation (RZCC) algorithm, which greatly increases the peak value of the main peak. In addition, plenty of researchers focus on the tracking problem of the BOC signal $[4,9,13,22,23]$. Many scholars have studied the BOC signal multipath elimination technology [24-27], which can be mainly categorized into nonparametric and parameterized class [26]. The CCRW method is applied to the $\operatorname{BOC}(1,1)$ signal in Reference [24]. Wu and Dempster propose the "BOC-Gated-PRN" method [25]. Zhao et al. propose an improved CCRW method for BOC signals with odd modulation coefficient [26]. Another class of the multipath mitigation technique is the parameterization method such as that in [27], where the authors propose an averaging-FFT method, which achieves robustness for both low-order and high-order BOC signals well.

Among the existing acquisition algorithms, some need to use filters, which are complex and easy to cause correlation loss, such as the Fishman method and Martin method. Some algorithms need to construct auxiliary signals, which increase the computational complexity, such as those in References
$[3,10]$. Some cannot be applied to all BOC $(m, n)$ signals. For instance, literature [9] is suitable for the $\sin$-BOC $(n, n)$ signal, and literature [3] is applicable for the sin-BOC $(k n, n)$ signal. Therefore, it is necessary to research on an algorithm which can be applied to all BOC signals with low complexity.

\section{Analysis of Existing Algorithms}

The filter bandwidth of the Martin method includes two principal lobes and all the secondary lobes between the principal ones. The filtered signal is correlated with the local PRN code, which is shifted and kept at the same rate as the received signals. The flow chart of the Martin method is shown in Figure 1, where parameter $a$ of the shift factor is determined by Formula (1). Since the Martin method is less complex than the Fishman method, it is selected as the representative of the BPSK-like method for analysis in this paper.

$$
a=\frac{N_{\mathrm{BOC}}}{2} .
$$

ASPeCT uses the PRN code as an auxiliary signal, and it can be applied to $\sin$-BOC $(n, n)$. On the other hand, GRASS is suitable for $\sin$-BOC $(k n, n)$ while SCPC is applicable for BOC $(m, n)$. Therefore, we choose SCPC for analysis because of its wider applicability. SCPC shifts the phase of the BOC signal by $\pi / 2$ to obtain the quadrature BOC signal (QBOC) and then uses QBOC as an auxiliary signal to eliminate the correlation peak ambiguity. The SCPC method in Reference [12] uses an ideal filter with a bandwidth of $2\left(f_{m}+f_{n}\right)$ to filter the received signal. The flow chart of the SCPC method is shown in Figure 2, and the final decision function is shown as

$$
R_{\mathrm{SCPC}}(\tau)=R_{\mathrm{BOC} / \mathrm{QBOC}}^{2}(\tau)+R_{\mathrm{BOC}}^{2}(\tau) .
$$

The RZCC method captures the BOC signal by folding ACF, which can effectively improve the correlation peak value of ACF. The main idea of RZCC is as follows: the received signal is divided into $I$ and $Q$ branches, which are 


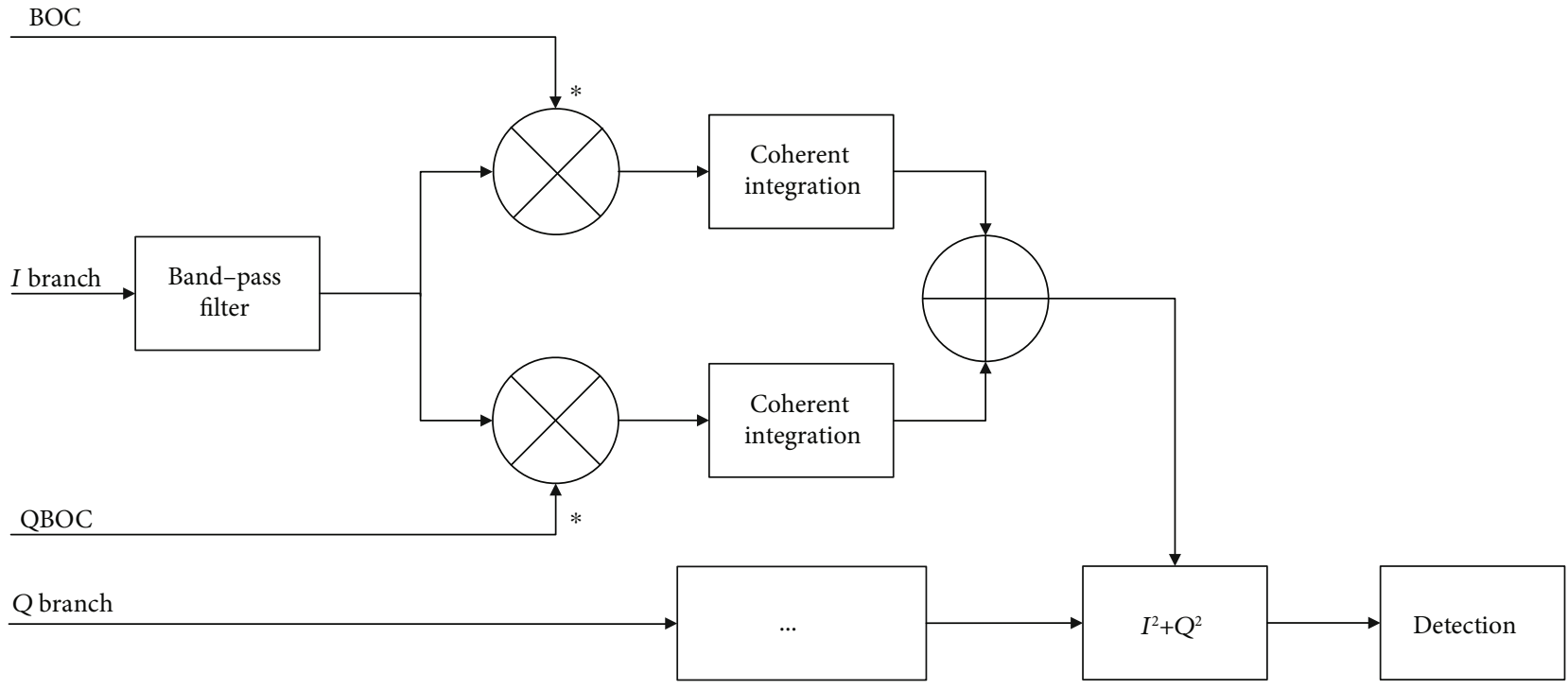

FIGURE 2: Flow chart of the SCPC method.

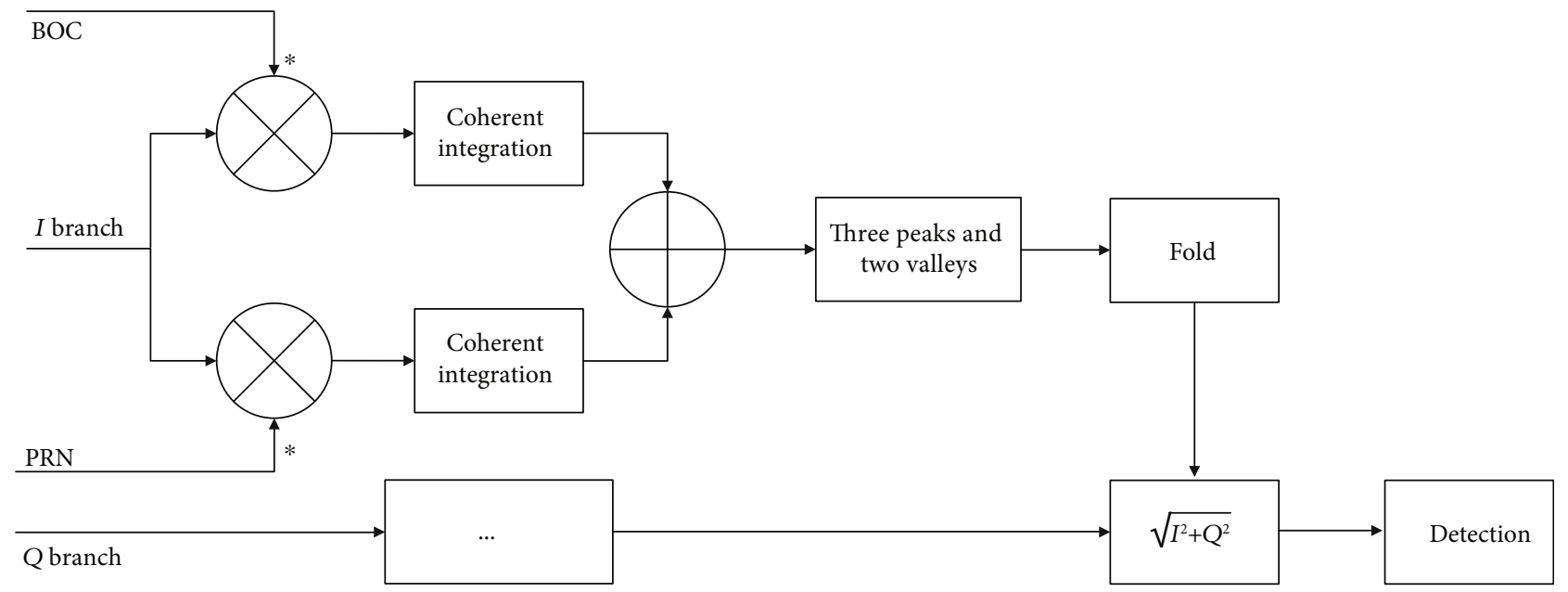

Figure 3: Flow chart of the RZCC method.

correlated with the local PRN code and the local BOC code, respectively; then, $\left|R_{\mathrm{BOC}}\right|$ and $\left|R_{\mathrm{BOC} / \mathrm{PRN}}\right|$ are obtained. For $\left|R_{\mathrm{BOC}}\right|+\left|R_{\mathrm{BOC} / \mathrm{PRN}}\right|$, three peaks and two valleys are searched, respectively, and then, the final ACF is obtained by enfoldment. The flow chart of the RZCC method is shown in Figure 3.

As shown in Figure 4, $I A B-B C D-D E J$, where $A, C$, and $E$ are the three peaks while $B$ and $D$ are the two valleys, is the initial ACF. In the first enfoldment, $A F K-K G E$ and $A B C-C$ $D E$ are symmetrical about line 1 . Hence, the coordinates of $F$ can be expressed as

$$
\begin{aligned}
& x_{F}=x_{B}, \\
& y_{F}=2 y_{A}-y_{B} .
\end{aligned}
$$

The coordinates of $G$ and $K$ can be determined with the same method. After the first enfoldment, ACF becomes
$I F K-K G J$. In the second enfoldment, $F H G$ and $F K G$ are symmetrical about line 2. After the second enfoldment, ACF turns into IFHGJ, which is the final ACF, as shown in Figure 4(d).

The RZCC algorithm is prone to frequency capture errors. It is because the RZCC algorithm folds the $I$ branch and the $Q$ branch separately and then calculates $\sqrt{I^{2}+Q^{2}}$. If the peaks of the $I$ and $Q$ branches are not at the same point and the energy of the $I$ and $Q$ branches is similar (an example is given in Figures 5(a) and 5(b)), the peak points of the $I$ and $Q$ branches appear at 885 and 905 , respectively. The result shows that the gain brought by enfoldment will not be very large. In this case, the peak after enfoldment of the adjacent frequency may exceed the correct frequency, which leads to a frequency capture error.

For example, when $C / N_{0}=40 \mathrm{~dB} \cdot \mathrm{Hz}$, the Doppler frequency shift is $0 \mathrm{~Hz}$, the initial code phase is 905 chips, the IF is $50 \mathrm{MHz}$, and the sampling rate is $400 \mathrm{MHz}$; the 


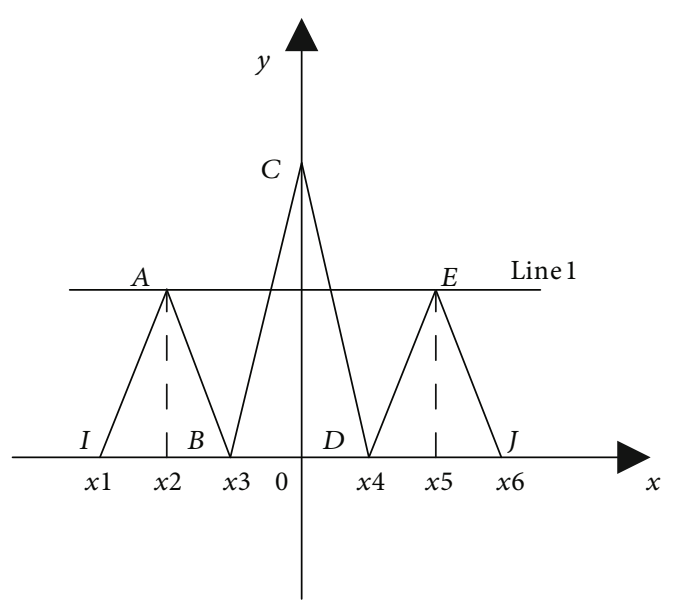

(a)

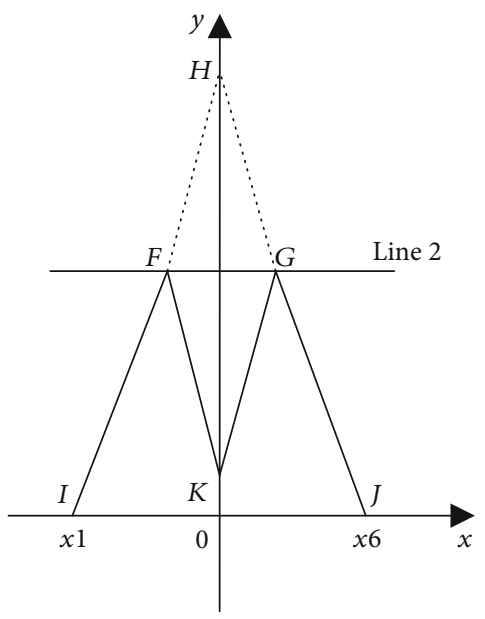

(c)

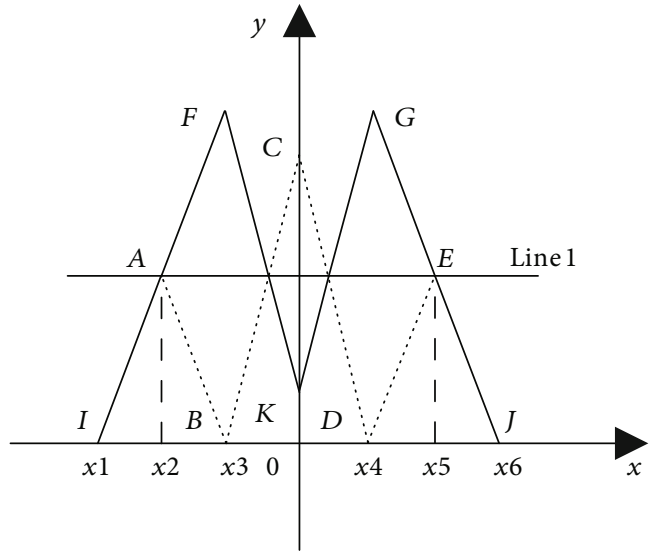

(b)

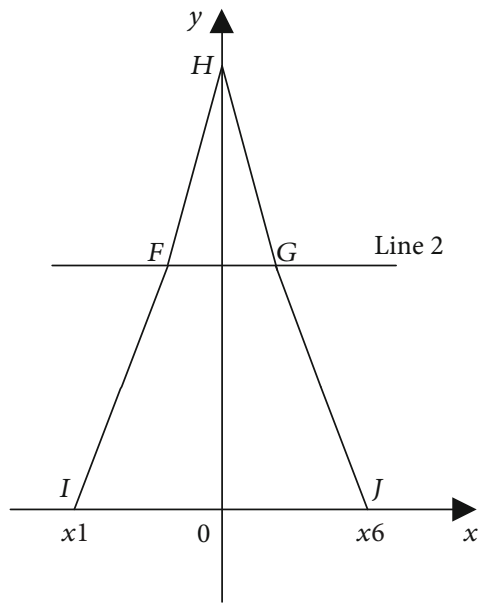

(d)

FIGURE 4: Principle of enfoldment.

sin-BOC $(10,5)$ signal is simulated. Figure 5 shows the results of the correct frequency. Figures 5(a) and 5(b) are the results of $I$ and $Q$ branches before enfoldment. Figure 5(c) is the correlation results after enfoldment, and Figure 5(d) is a partial enlarged view of Figure 5(c). The peak points of $I$ and $Q$ branches are at 885 and 905 with similar energy, respectively, but not at the same location. Figure 6 shows the results of adjacent frequency. The peak points of $I$ and $Q$ branches are at 905 and 361571, respectively. Although they are not at the same location, peak 361571 is much smaller than peak 905. Since the peak of adjacent frequency after enfoldment is $4.693 e 5$ while the correct frequency is $4.097 e 5$, the false acquisition of frequency will occur according to the RZCC capture criterion.

\section{Similar Enfoldment Acquisition Algorithm}

The SEA algorithm is an improved algorithm for the RZCC algorithm. In this paper, two kinds of BOC signal acquisition algorithms are proposed, which are the similar enfoldment acquisition with the quadratic threshold (SEAQT) algorithm and similar enfoldment acquisition with the single threshold (SEAST) algorithm.
The SEAQT flow chart is shown in Figure 7, where the signal process is divided into $I$ and $Q$ channels and the local reference code is the BOC signal. After the received signal correlation with $I$ and $Q$ branches, $I^{2}+Q^{2}$ is calculated. The SEAQT method makes the first threshold decision for $I^{2}+Q^{2}$, and the decision threshold $\eta_{1}$ equals to $\alpha * \eta_{0}$, where $\eta_{0}$ corresponds to the capture threshold for the ambiguous BOC method, $0 \leq \alpha \leq 1$. If the capture statistic exceeds threshold $\eta_{1}$, it is folded to determine whether it exceeds threshold $\eta_{2}$. If the capture statistic exceeds threshold $\eta_{2}$, the signal is considered to be captured.

Unlike RZCC, the SEAQT method does not require a PRN code as an auxiliary signal which applies two threshold decisions to determine whether signal exists or not.

The flow chart of SEAST is shown in Figure 8. SEAST searches for three peaks and two valleys of capture statistic and then folds them and makes a decision.

3.1. Comparison between SEA and RZCC. There are two main differences between the SEA algorithm and RZCC: (1) SEA calculates $I^{2}+Q^{2}$ first and folds later. (2) The SEA does not require a PRN code as an auxiliary signal. 


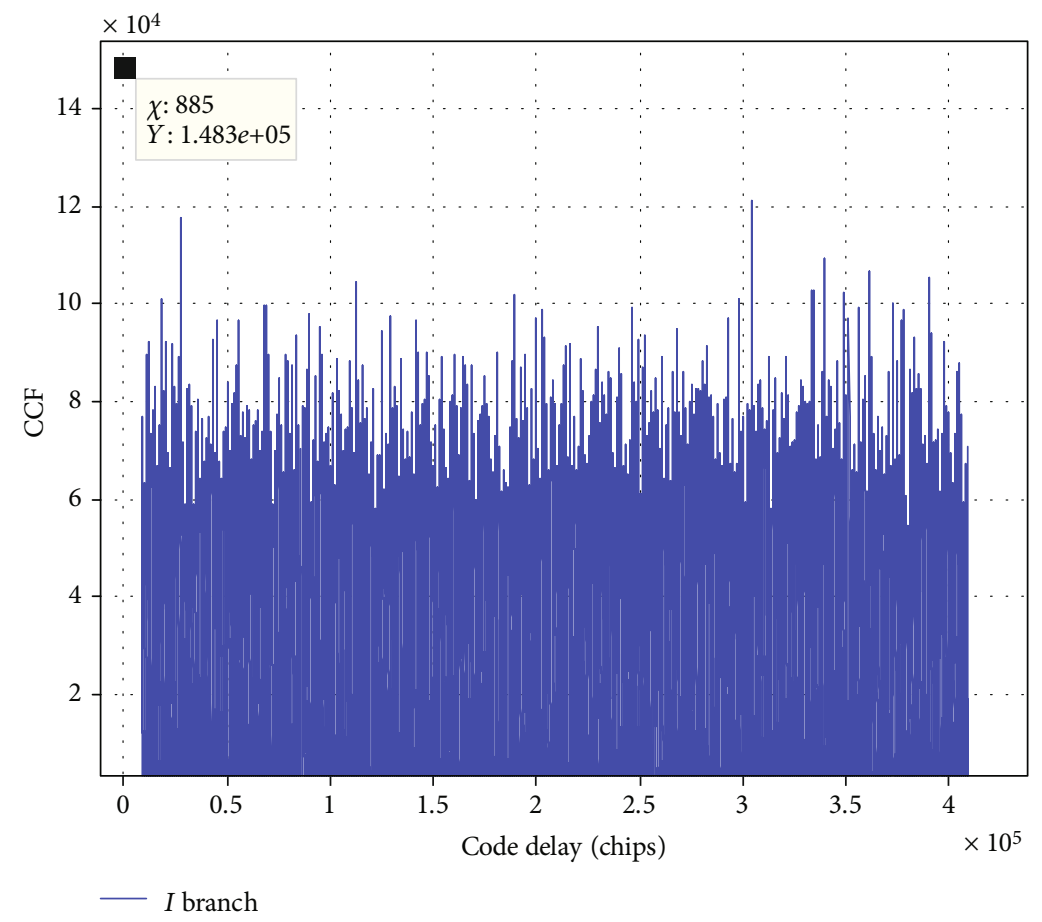

(a) Result of $I$ branch before enfoldment

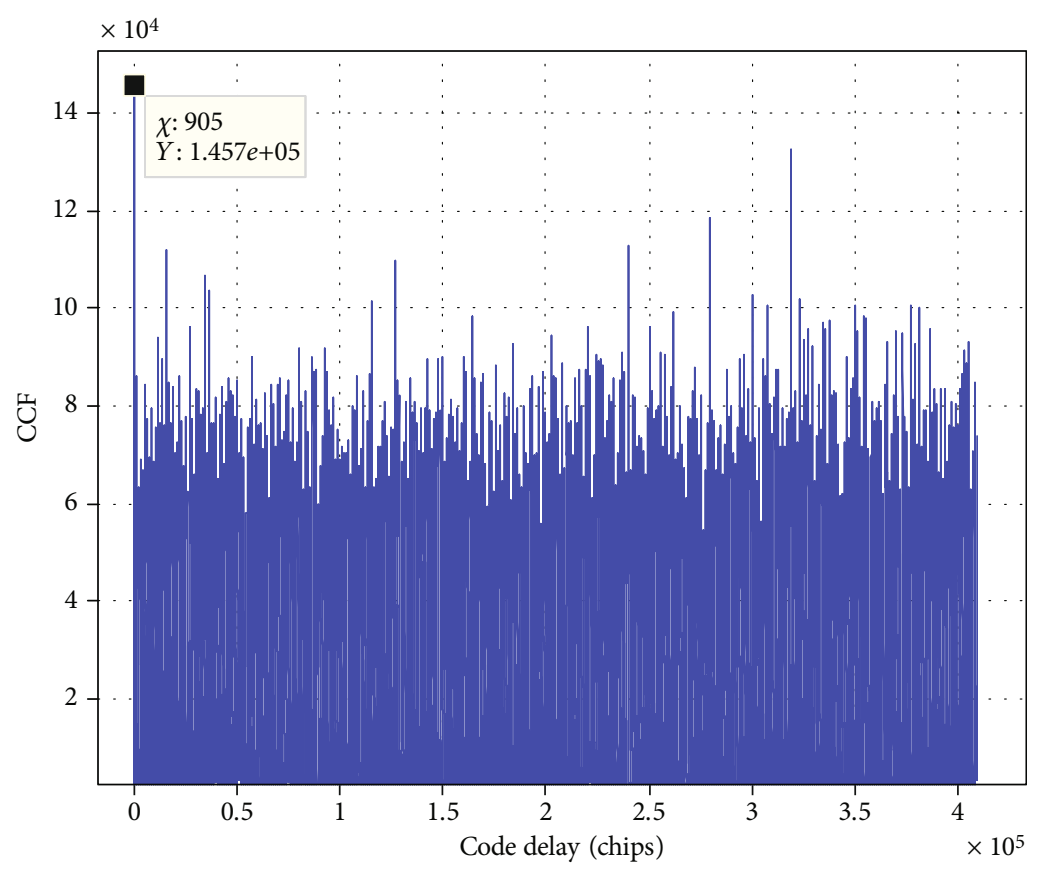

- Q branch

(b) Result of $Q$ branch before enfoldment

FIgUre 5: Continued. 


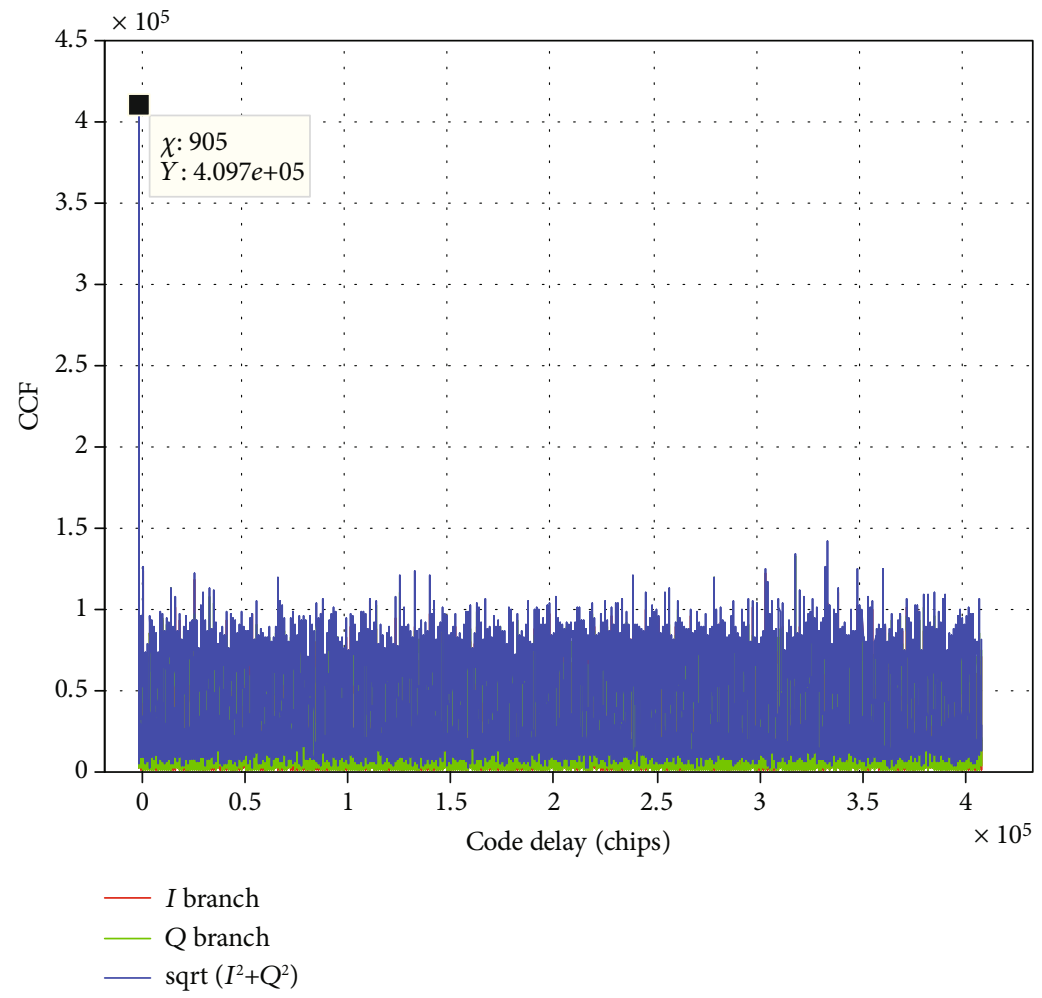

(c) Results after enfoldment

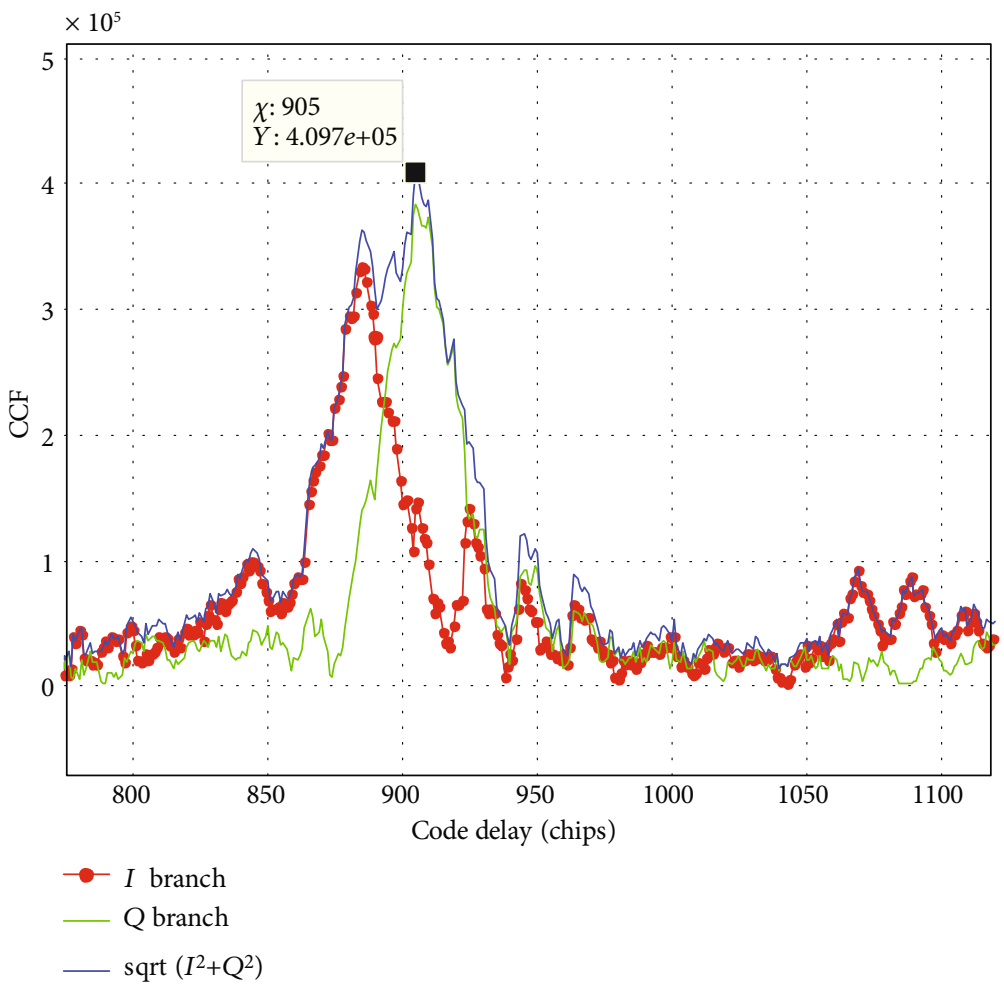

(d) Partial enlarged view of (c)

Figure 5: Correlation results of the correct frequency.

Still using the example of Figures 5 and 6, I branch, $Q$ branch, and $\sqrt{I^{2}+Q^{2}}$ results before the enfoldment of the correct frequency are shown in Figure 9(a), and Figure 9(b) is a partial enlarged view of Figure 9(a). As can be seen in Figure 9(b), the peaks of $I$ branch, $Q$ branch, and $\sqrt{I^{2}+Q^{2}}$ are all at 905 chips. After enfoldment, $\sqrt{I^{2}+Q^{2}}$ is shown in 


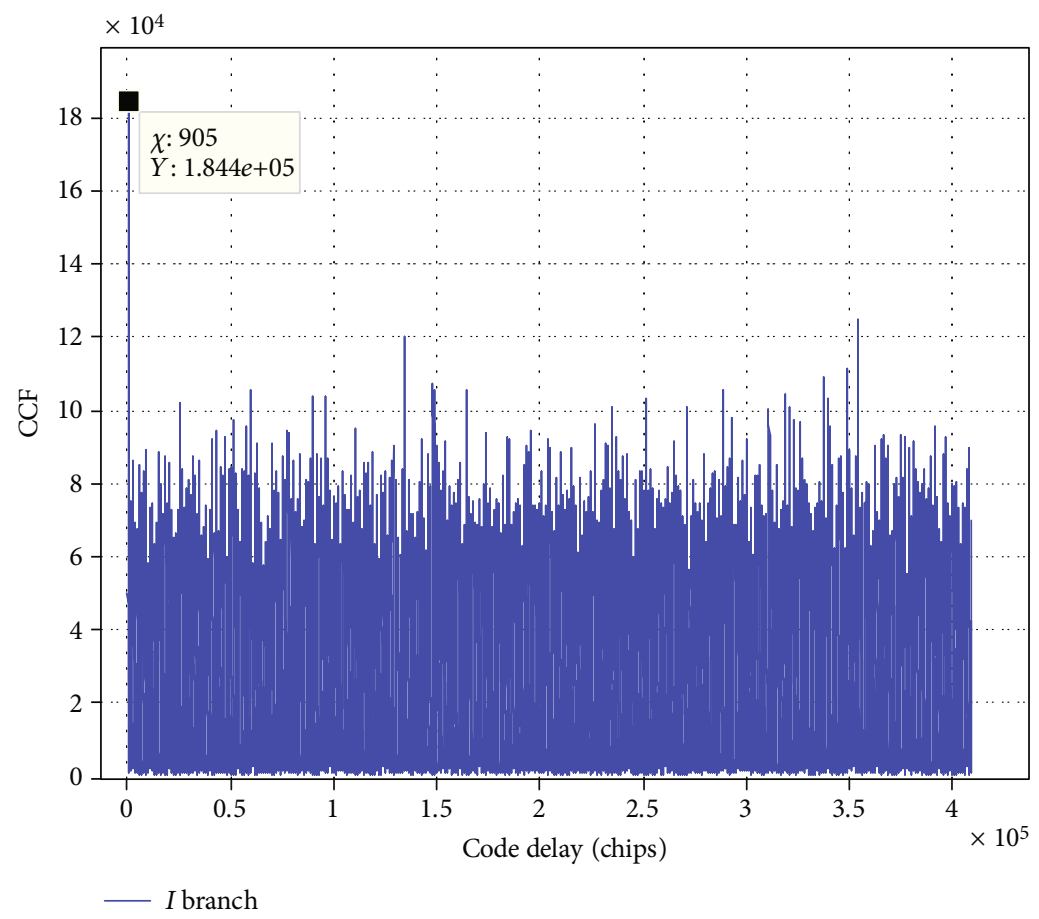

(a) Result of $I$ branch before enfoldment

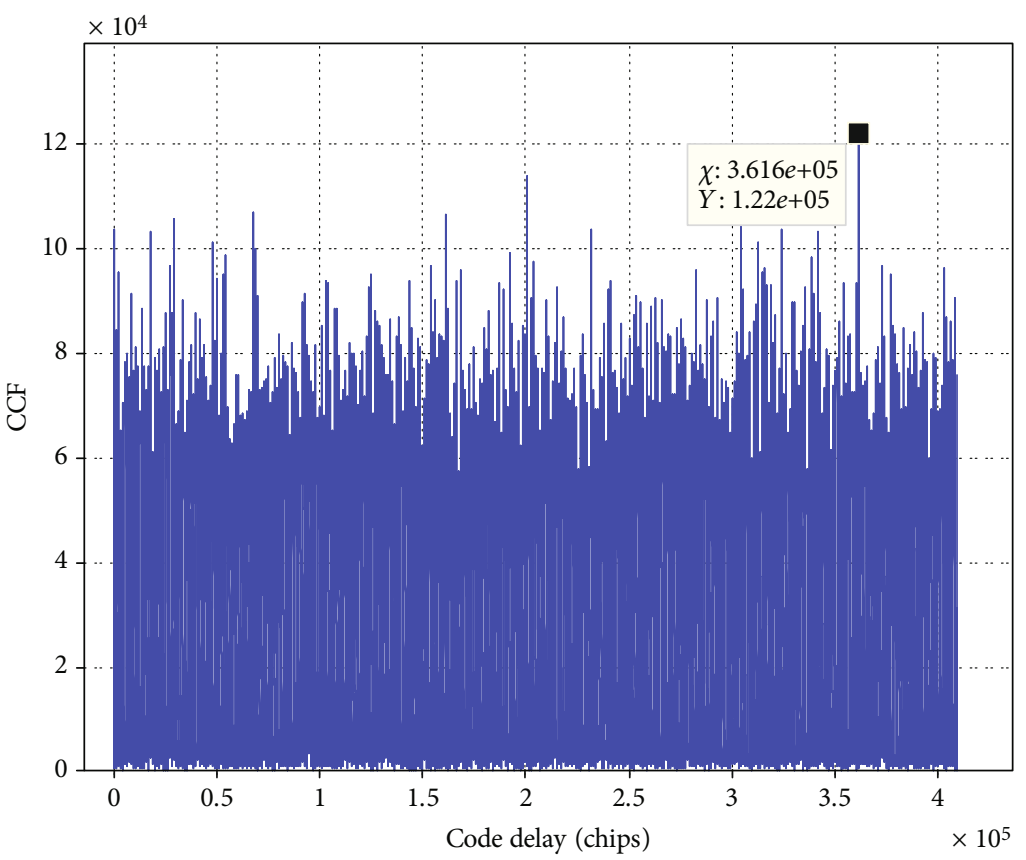

- Q branch

(b) Result of $Q$ branch before enfoldment

Figure 6: Continued. 


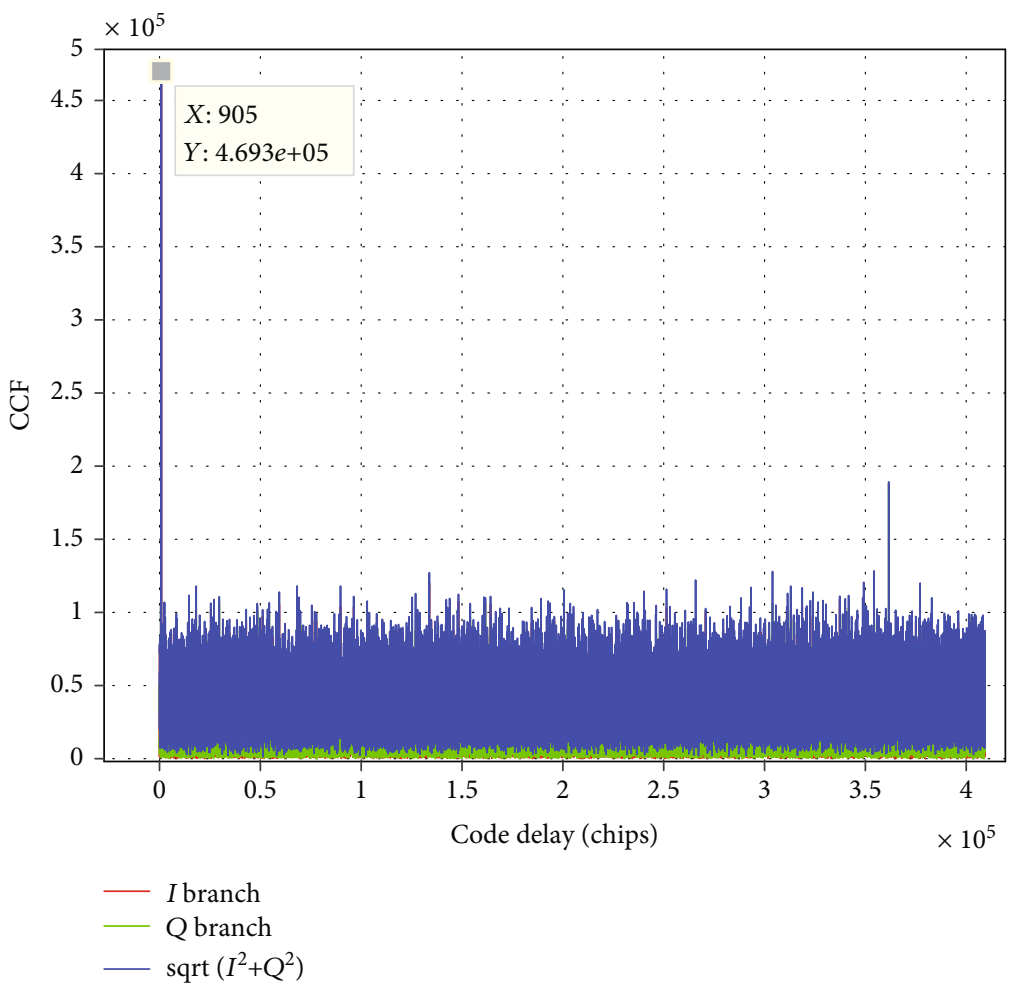

(c) Results after enfoldment

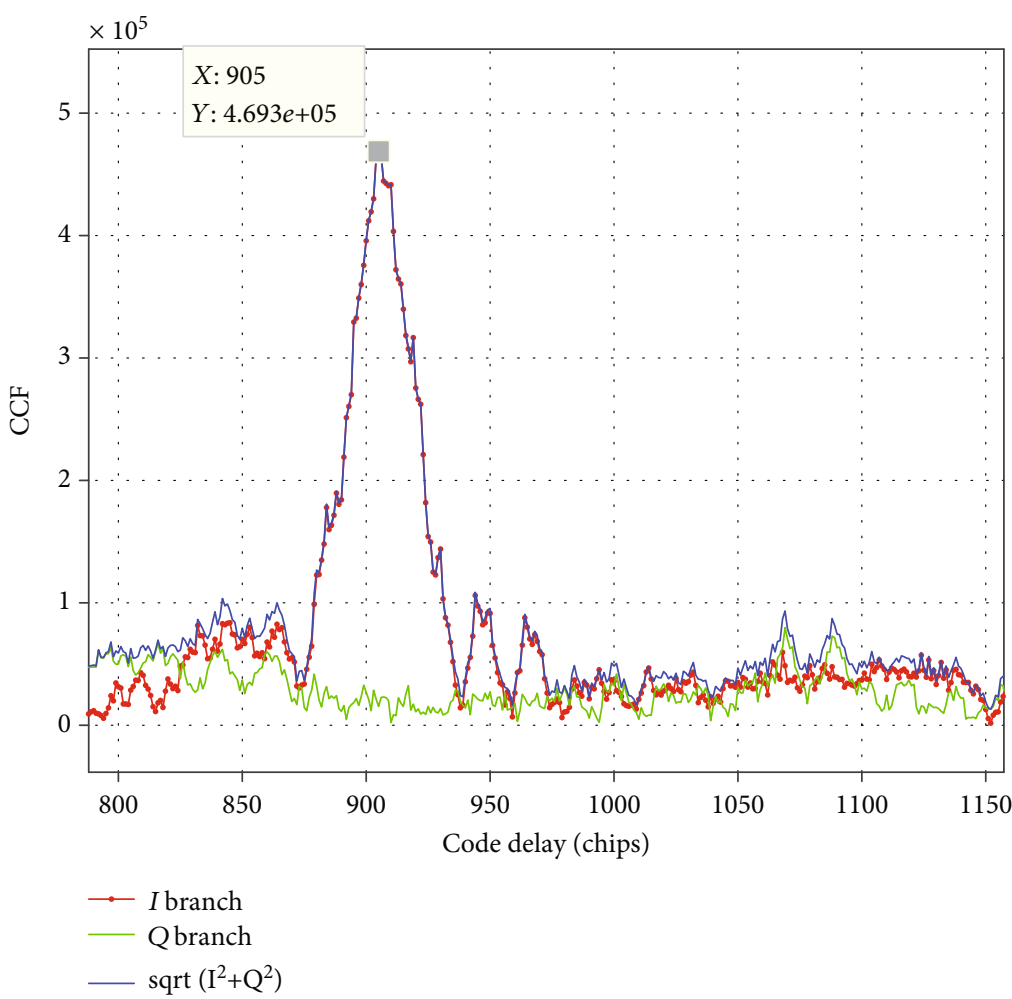

(d) Partial enlarged view of (c)

Figure 6: Correlation results of the adjacent frequency.

Figure 10. It can be seen from the figure that the correlation peak corresponding to the correct frequency exceeds the left and right adjacent frequencies, which avoids false detection.
3.2. Enfoldment Gain of SEA. For the ambiguous BOC method, when the modulation order is $M$ and the value of the main peak is normalized to 1 , as a result, the second peak 


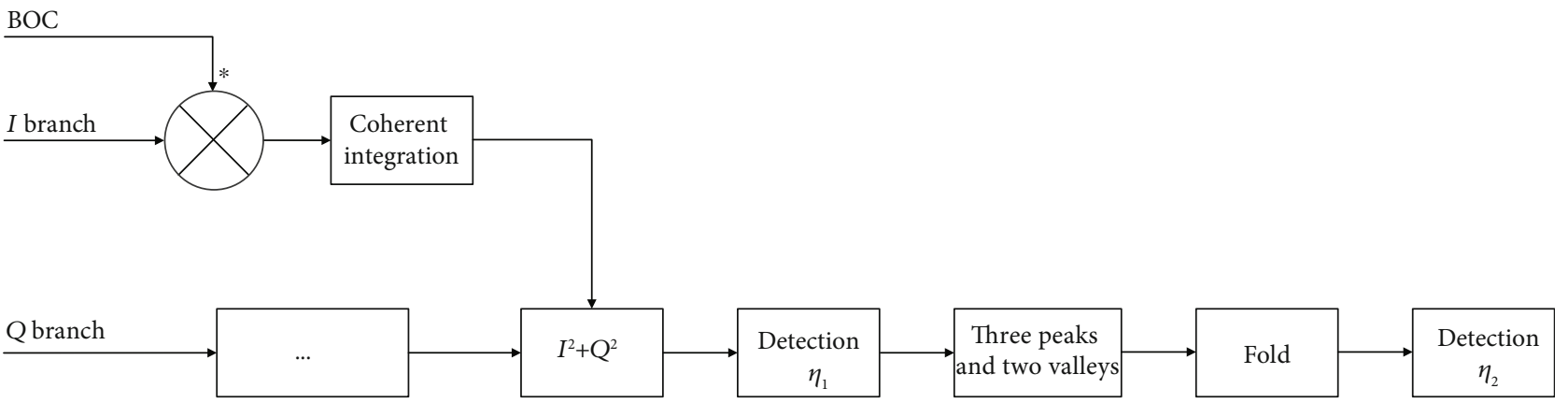

FIgURE 7: Flow chart of the SEAQT method.

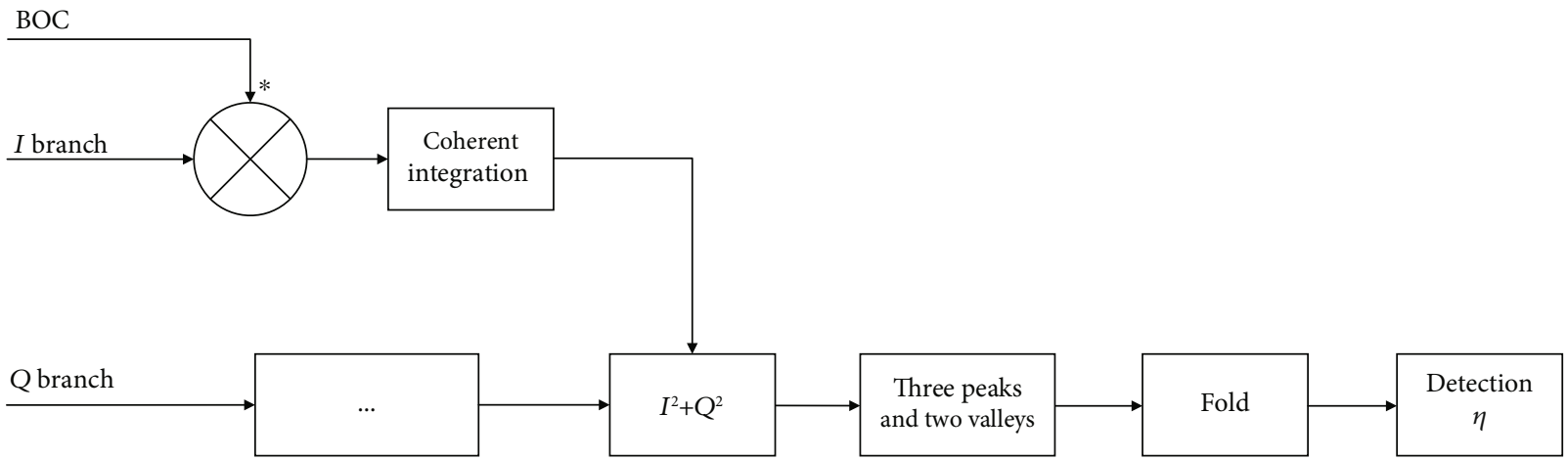

Figure 8: Flow chart of the SEAST method.

value is $((M-1) / M)^{2}$. Therefore, the main peak of capture statistic is $10 \lg \left(1 /((M-1) / M)^{2}\right)=20 \lg ((M-1) / M) \mathrm{dB}$ stronger than the second peak. For instance, when the modulation order $M=2$, the main peak is $6 \mathrm{~dB}$ stronger than the second peak. When the modulation order $M=4$, the main peak is $2.5 \mathrm{~dB}$ stronger than the second peak.

The enfoldment process of the SEA method is shown in Figure 11.

The ordinates of points $A$ and $E$ are

$$
y_{A}=y_{E}=\frac{(M-1)^{2}}{M^{2}} \text {. }
$$

Points $B$ and $B^{\prime}$ and $D$ and $D^{\prime}$ are symmetrical about line 1 . According to the principle of symmetry, the ordinates of $B^{\prime}$ and $D^{\prime}$ are

$$
y_{B^{\prime}}=y_{D^{\prime}}=2 y_{A}-y_{B}=\frac{2(M-1)^{2}}{M^{2}} .
$$

Similarly, the ordinate of point $C^{\prime}$ is

$$
y_{C^{\prime}}=2 y_{A}-y_{C}=\frac{2(M-1)^{2}}{M^{2}}-1 .
$$

Point $C^{\prime \prime}$ is the maximum correlation peak. Similarly, the ordinate of point $C^{\prime \prime}$ is

$$
y_{C^{\prime \prime}}=2 y_{B^{\prime}}-y_{C^{\prime}}=\frac{2(M-1)^{2}+M^{2}}{M^{2}} .
$$

When $M \geq 2$, the third peak is $((M-2) / M)^{2}$. Therefore, the main peak is $10 \lg \left(\left(2(M-1)^{2}+M^{2}\right) /(M-2)^{2}\right) \mathrm{dB}$ stronger than the third peak.

$10 \lg \left(\frac{\left(2(M-1)^{2}+M^{2}\right) / M^{2}}{((M-2) / M)^{2}}\right)=10 \lg \left(\frac{2(M-1)^{2}+M^{2}}{(M-2)^{2}}\right) \mathrm{dB}$.

Compared with the ambiguous BOC method, the ratio of the main peak to the secondary peak increases:

$$
\begin{aligned}
10 \lg & \left(\frac{2(M-1)^{2}+M^{2}}{(M-2)^{2}}\right)-20 \lg \left(\frac{M}{M-1}\right) \\
& =10 \lg \left(\frac{2(M-1)^{2}+M^{2}}{(M-2)^{2}} \cdot \frac{(M-1)^{2}}{M^{2}}\right) \mathrm{dB} .
\end{aligned}
$$

When the modulation order $M=4$, the main peak is $9.3 \mathrm{~dB}$ stronger than the secondary peak, which is $6.8 \mathrm{~dB}$ higher than that of the ambiguous BOC method. 


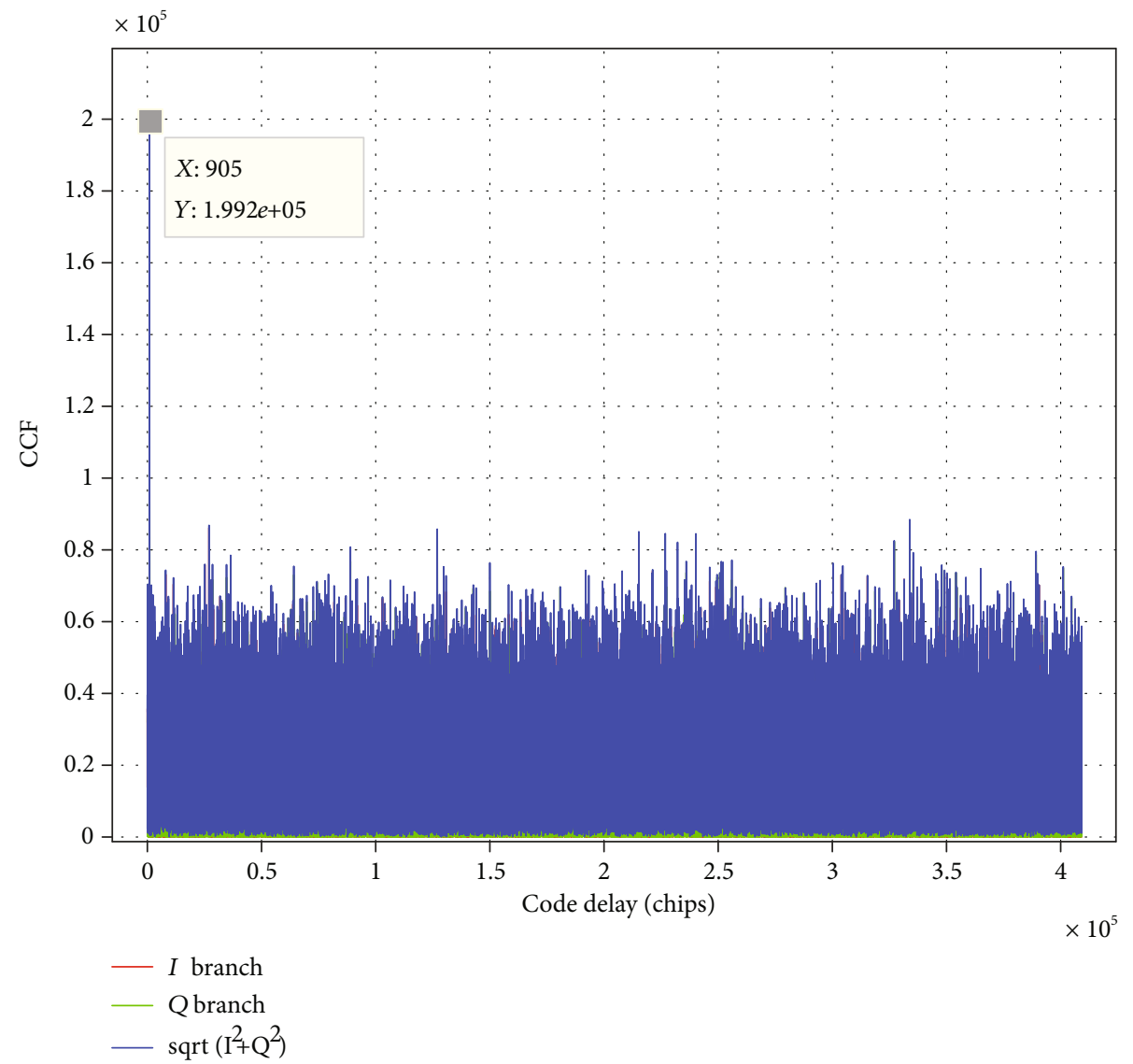

(a) Results before enfoldment

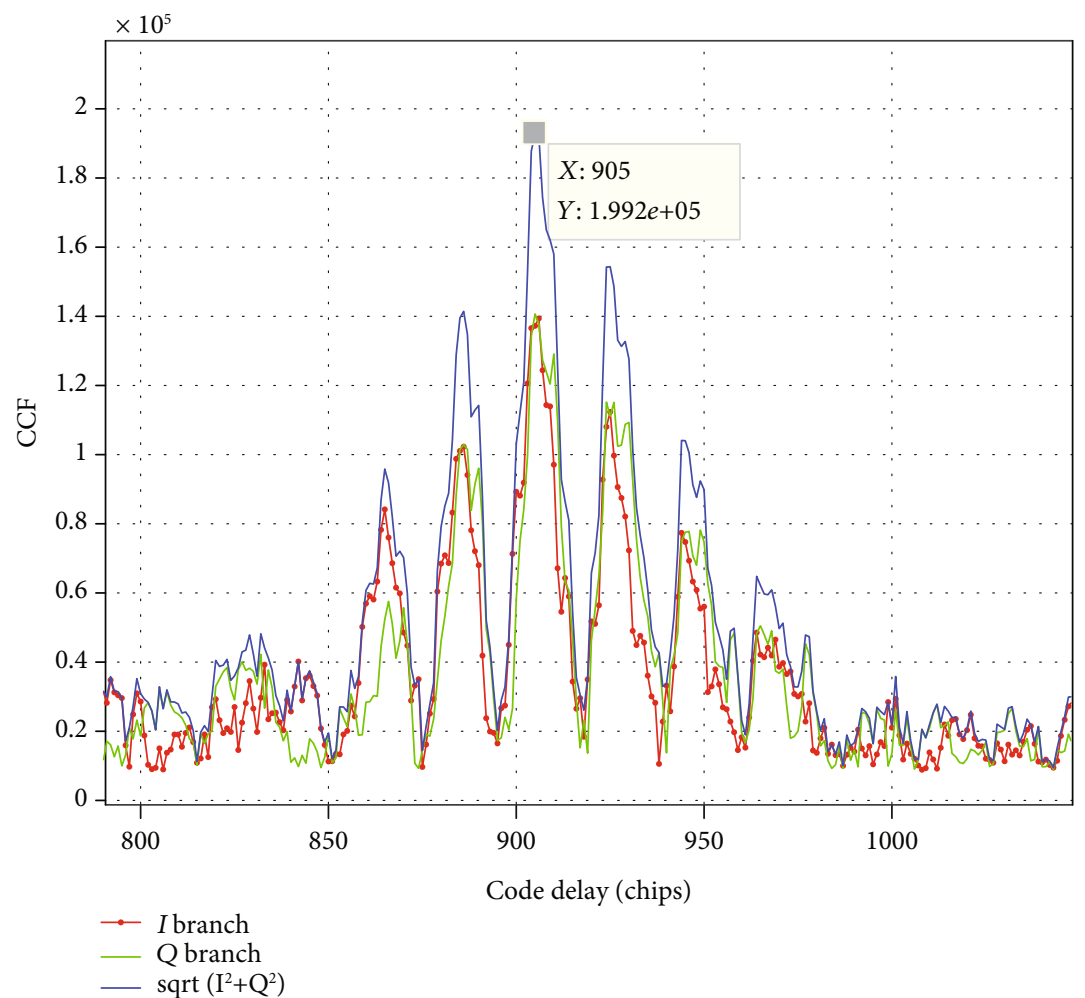

(b) Partial enlarged view of (a)

FIgURE 9: Results of the correct frequency. 


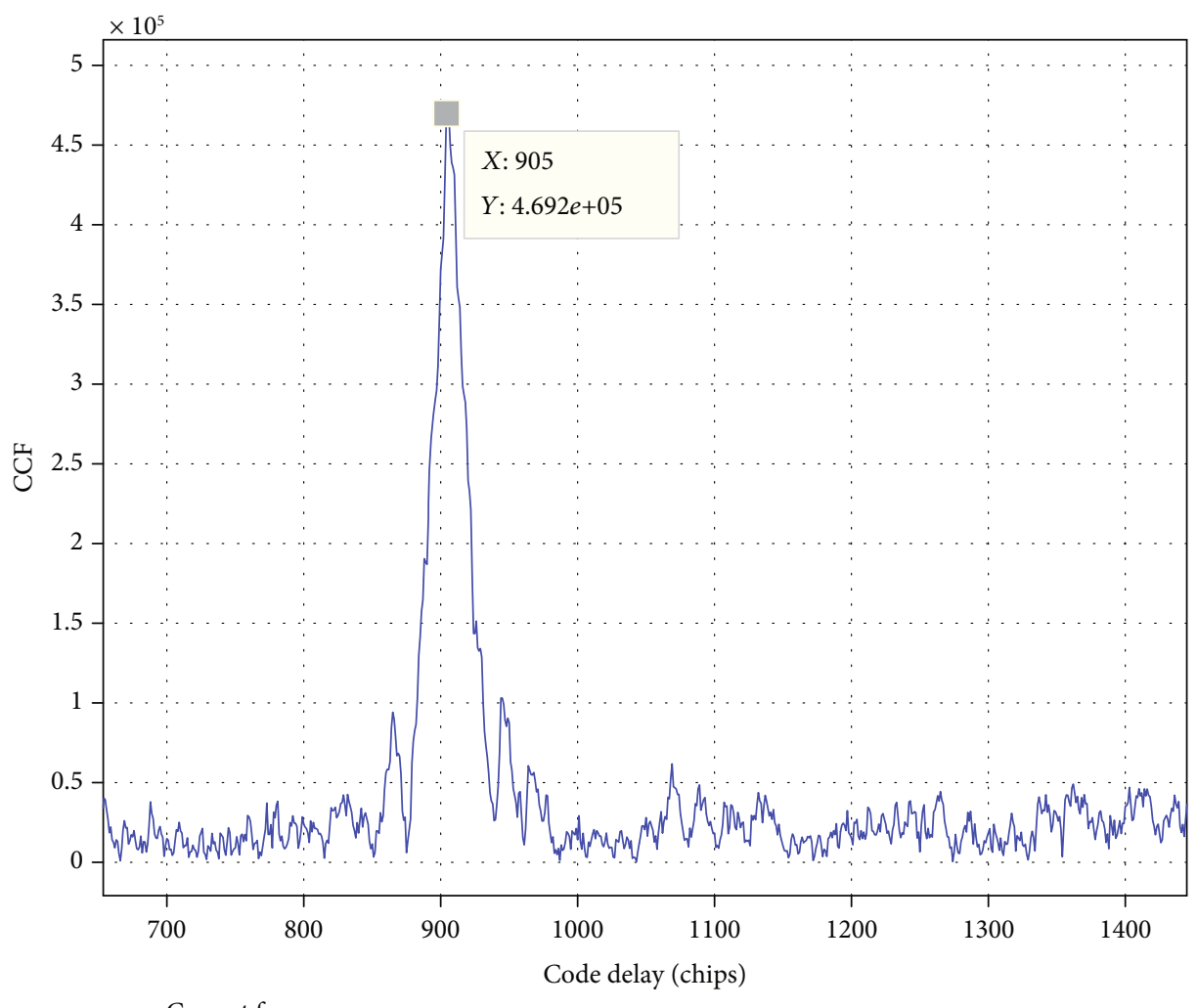

(a) $\sqrt{I^{2}+Q^{2}}$ of correct frequency

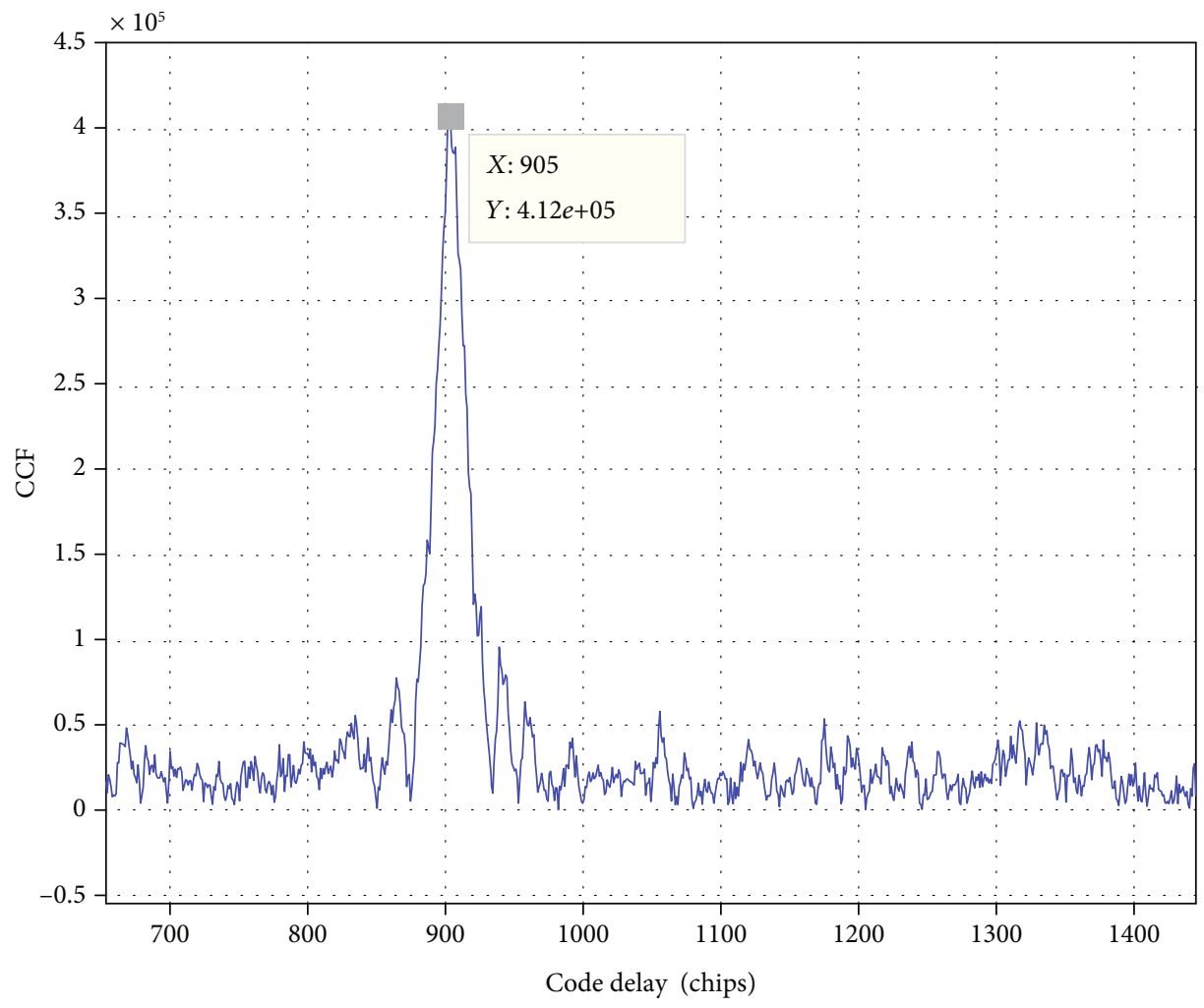

- Adjacent frequency 1

(b) $\sqrt{I^{2}+Q^{2}}$ of adjacent frequency 1

Figure 10: Continued. 


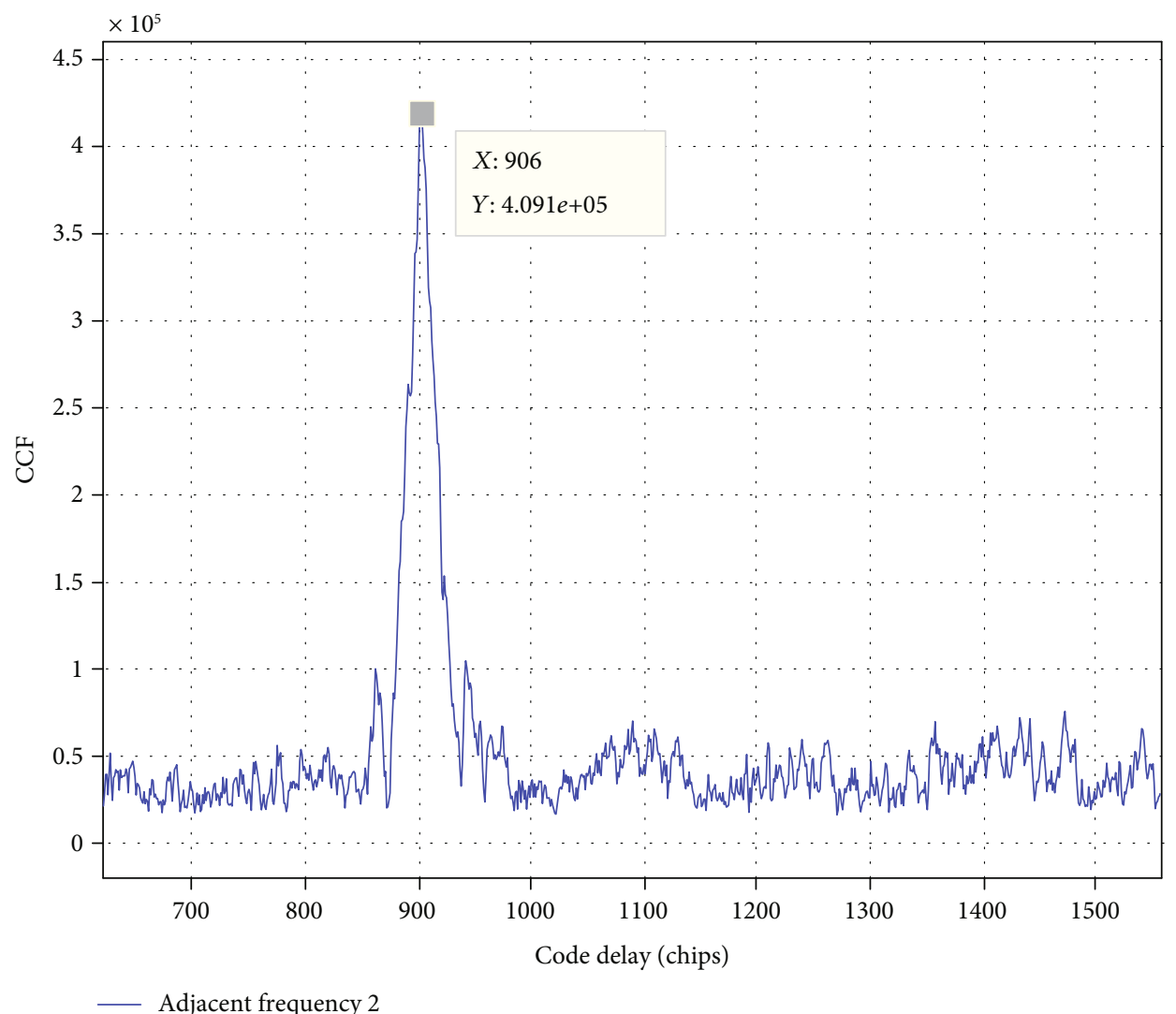

(c) $\sqrt{I^{2}+Q^{2}}$ of adjacent frequency 2

Figure 10: $\sqrt{I^{2}+Q^{2}}$ after enfoldment of correct and adjacent frequencies.
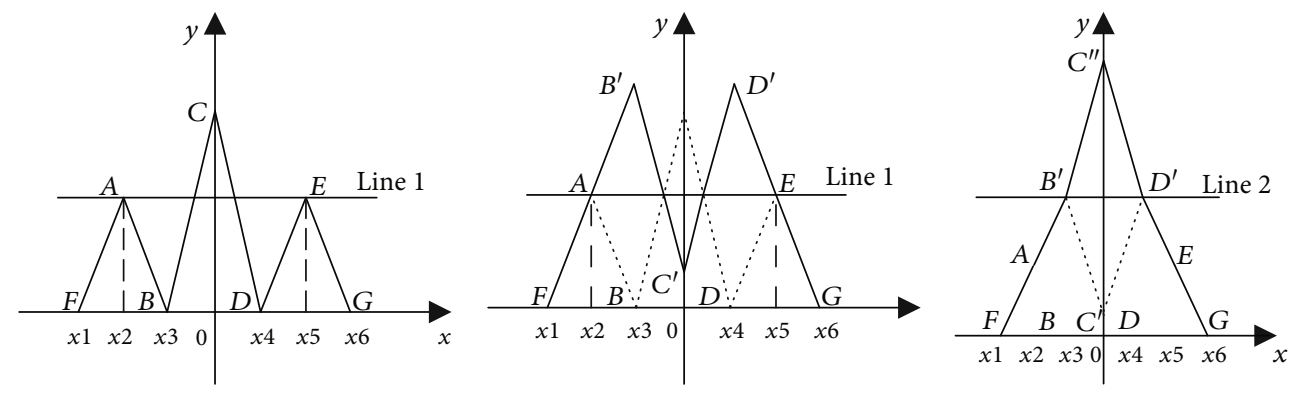

FIGURE 11: Enfoldment process of the SEA method.

\section{Performance Analysis}

4.1. Theoretical Analysis. The BOC signal broadcasted by the satellite can be expressed as

$$
s(t)=\sqrt{E_{b}} D(t) x_{P}(t) x_{B}(t) \sin \left(2 \pi f_{c} t+\theta\right) .
$$

Among them, $E_{b}$ is the signal energy, $D(t)$ is the data code broadcasted by the satellite, $x_{P}(t)$ is the PRN code, $x_{B}(t)$ is the BOC code, $f_{\mathrm{c}}$ is the carrier frequency, $\theta$ is the initial phase of the signal.
The signal after the downconverter and analog-to-digital converter (ADC) can be expressed as

$$
\begin{aligned}
s_{\mathrm{IF}}(n)= & A D(n-\tau) x_{P}(n-\tau) x_{B}(n-\tau) \sin \\
& \cdot\left(2 \pi\left(f_{\mathrm{IF}}+f_{d}\right) n+\theta\right)+\delta(n) .
\end{aligned}
$$

Among them, $A$ is the amplitude of the received signal, $f_{\text {IF }}$ is the intermediate frequency, $\delta(n)$ is the Gauss white noise with zero mean and the variance $\sigma^{2}, D(n-\tau)$ is the data code of the received signal, $x_{P}(n-\tau)$ is the PRN code 


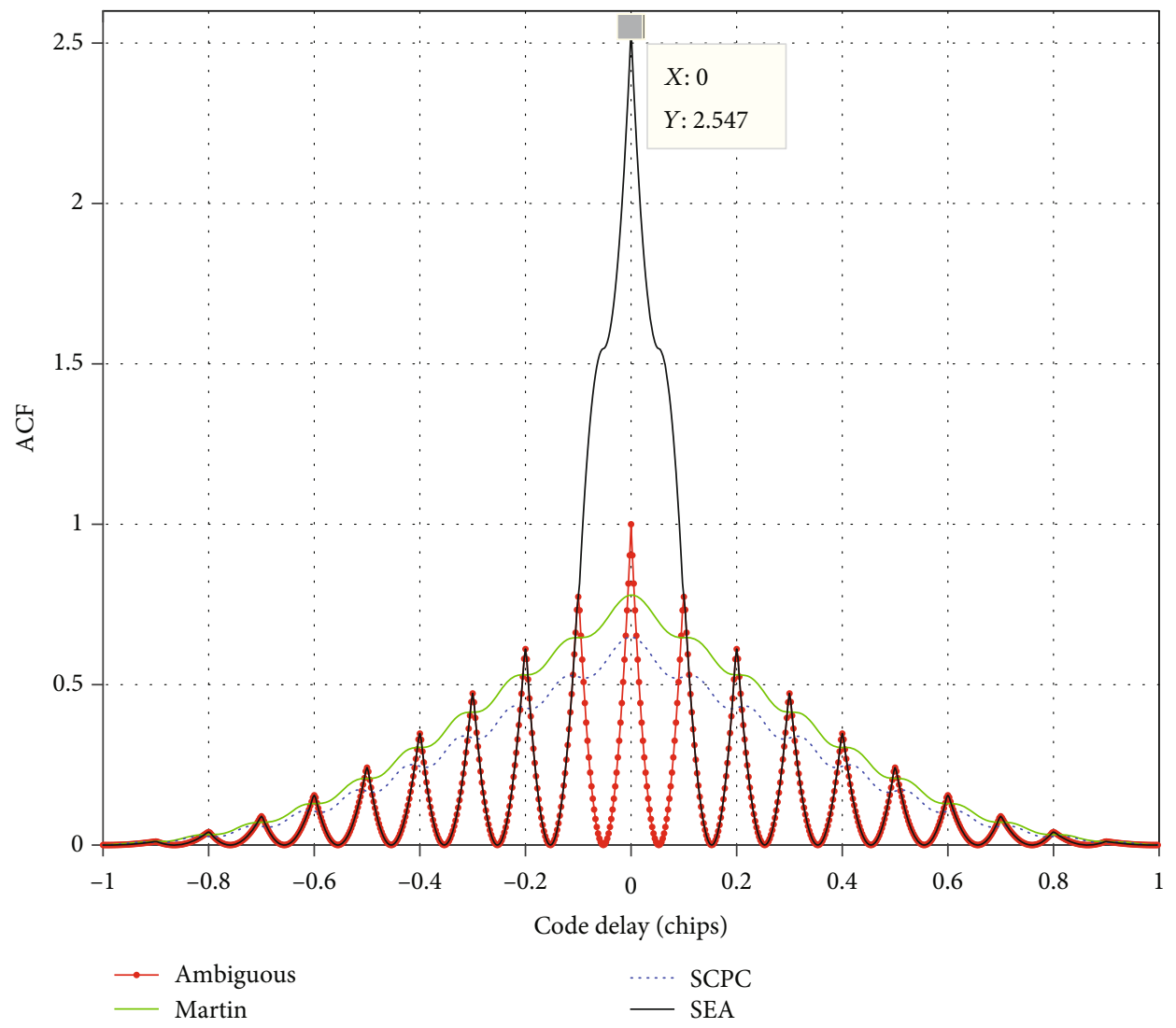

FIGURE 12: ACF of four methods.

of the received signal, and $x_{B}(n-\tau)$ is the BOC code of the received signal.

Detection statistic of the ambiguous BOC method is

$$
V=\frac{1}{L} \sum_{n=0}^{L-1} \sqrt{I^{2}(n)+Q^{2}(n)} .
$$

Among them, $I(n)$ and $Q(n)$ are in-phase components and quadrature components obtained by correlation between local codes and the received signals, respectively, and $L$ is the number of incoherent integrals. When the maximum correlation peak in detection $V$ exceeds the threshold, the signal is detected. $V_{t}, P_{\mathrm{md}}$, and $P_{\mathrm{fa}}$ refer to the capture threshold, the probability of missed detection, and the probability of false alarm, respectively.

The false alarm probability $P_{\mathrm{fa}}$ corresponding to the capture threshold $V_{t}$ is

$$
P_{\mathrm{fa}}=\int_{V_{t}}^{\infty} \frac{v}{\sigma^{2}} e^{-\left(v^{2} / 2 \sigma^{2}\right)} d v=e^{-\left(V_{t}^{2} / 2 \sigma^{2}\right)} .
$$

Thus, the capture threshold $V_{t}$ is

$$
V_{t}=\sigma \sqrt{-2 \ln P_{\mathrm{fa}}}
$$

In this paper, the ambiguous BOC method, Martin method, SCPC method, and SEA method are selected for simulation verification. At the same time, the performance of RZCC and SEA is compared and analyzed. For the sinBOC $(10,2)$ signal, the ACF of the four algorithms is shown in Figure 12. The maximum correlation peak of the ambiguous BOC algorithm is normalized to 1. Because of filtering, the maximum peak of the Martin method and SCPC method is less than 1. Since the modulation order of the sin-BOC $(10,2)$ signal is 10 , according to Formula $(7)$, the maximum correlation peak of the SEA method is 2.62. As shown in Figure 12, the maximum correlation peak of SEA is 2.55, which is in accordance with the theoretical analysis results.

4.2. Applicability Analysis of the SEA Algorithm. The SEA algorithm applies the ACF similarity of the BOC signal. Whether it is the sin-BOC signal or cos-BOC signal, for the BOC signal of any modulation order, the adjacent two secondary peaks of the BOC signal ACF have similarities with the main peak. Therefore, the enfoldment method can be utilized. In other words, SEA is suitable for sin-BOC and cos-BOC signals of any order in theory.

Four kinds of BOC signals, sin-BOC $(10,5)$, cos-BOC $(1,1)$, sin-BOC $(15,10)$, and cos-BOC $(10,4)$, are simulated with a sampling rate of $400 \mathrm{MHz}$, an IF of $30 \mathrm{MHz}$, a Doppler shift of $300 \mathrm{~Hz}$, and an initial code phase of 1500 chips (after sampling). When $C / N_{0}=40 \mathrm{~dB} \cdot \mathrm{Hz}$, the crosscorrelation function (CCF) of the local BOC signal and the received signal is shown in Figure 13. The ACF of the four BOC signals with no noise is shown in Figure 14. As can be seen from 


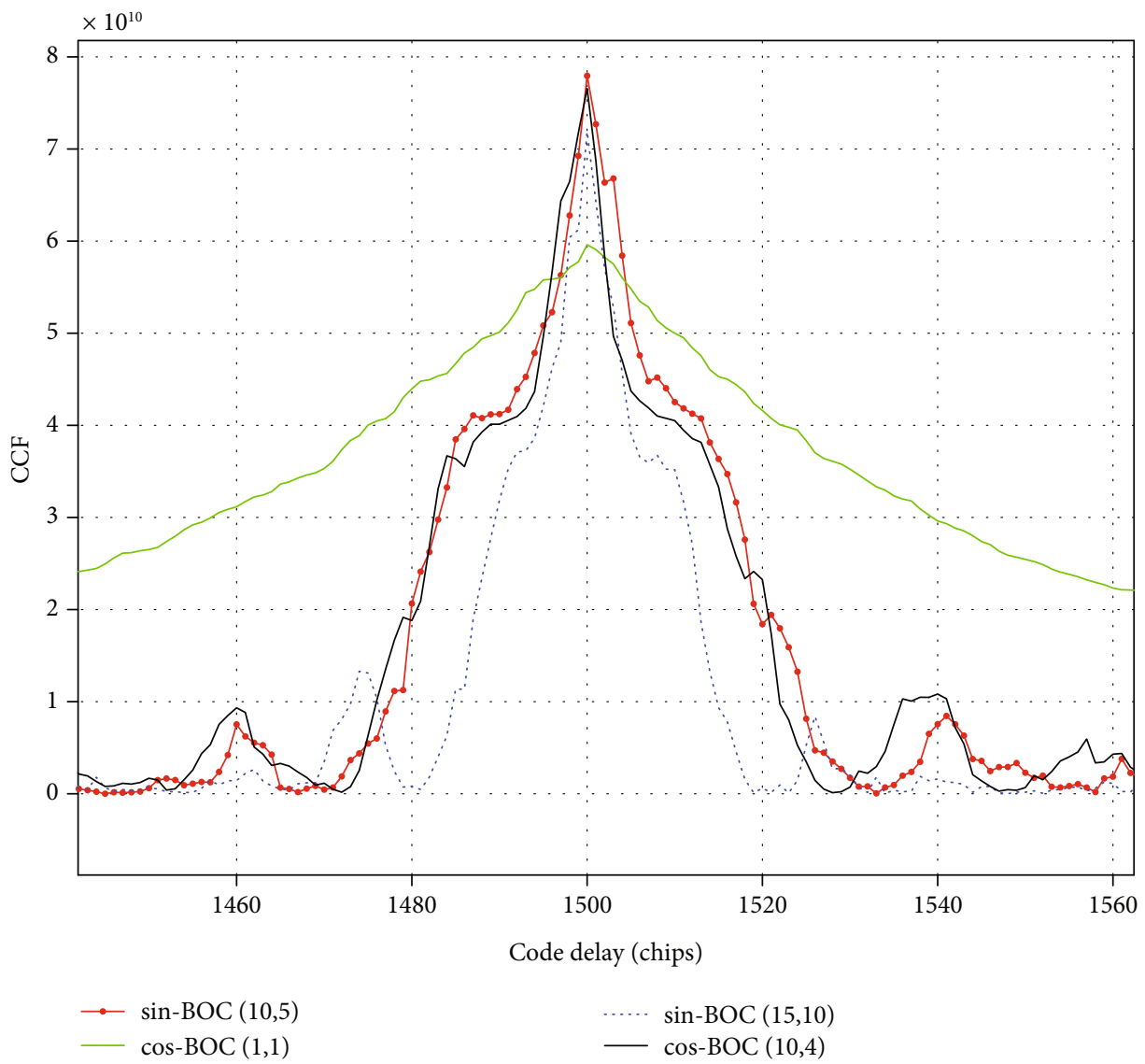

Figure 13: CCF of $C / N_{0}=40 \mathrm{~dB} \cdot \mathrm{Hz}$ modulated signal.

Figures 13 and 14, the SEA method is suitable for sin-BOC and cos-BOC signals with even or odd modulation orders.

4.3. Capture Performance Analysis. Correct detection probability $P_{\mathrm{cd}}$ and false detection probability $P_{\mathrm{fd}}$ are used to analyze capture performance. For BOC $(m, n)$ signals, the phase difference between the main peak and the second peak code is $f_{s} / 2 f_{n}$, where $f_{s}$ is the signal sampling rate. The captured code phase and frequency are recorded as $\varphi_{c}$ and $f_{c}$, respectively; the actual code phase and frequency are recorded as $\varphi_{\mathrm{a}}$ and $f_{\mathrm{a}}$, respectively.

The total number of Monte Carlo simulation is $N$. The number of correct detection $N_{0}$ is defined as the number of times, while the $\left|\varphi_{\mathrm{c}}-\varphi_{\mathrm{a}}\right|<f_{s} / 2 f_{n}$ and $f_{\mathrm{c}}=f_{\mathrm{a}}$ are simultaneously satisfied in the simulation. Consequently, the correct detection probability $P_{\mathrm{cd}}$ is defined as

$$
P_{\mathrm{cd}}=\frac{N_{0}}{N}
$$

The false detection number $N_{1}$ is defined as the number of times $\left|\varphi_{\mathrm{c}}-\varphi_{\mathrm{a}}\right| \geq f_{s} / 2 f_{n}$ or $f_{\mathrm{c}} \neq f_{\mathrm{a}}$ in the simulation. Therefore, false detection probability $P_{\text {fd }}$ is defined as

$$
P_{\mathrm{fd}}=\frac{N_{1}}{N}
$$

When the signal exists, if the capture statistic exceeds the capture threshold, the signal is considered to be detected, which corresponds to the detection probability $P_{\mathrm{d}}$. The opposite corresponds to the missed detection probability $P_{\mathrm{md}}$. Thus, the detection probability $P_{\mathrm{d}}$ and the missed detection probability $P_{\mathrm{md}}$ satisfy the following relationship.

$$
P_{\mathrm{d}}+P_{\mathrm{md}}=1
$$

Considering that the correct detection probability $P_{\mathrm{cd}}$ and the false detection probability $P_{\mathrm{fd}}$ in the paper are all defined under the condition that the signal is detected, the correct detection probability $P_{\mathrm{cd}}$ and the false detection probability $P_{\text {fd }}$ satisfy the following relationship.

$$
P_{\mathrm{cd}}+P_{\mathrm{fd}}=P_{\mathrm{d}}
$$

The first set of simulation conditions is set as follows: for the sin-BOC $(10,2)$ signal, the false alarm rate is $P_{\mathrm{fa}}=10^{-4}$, the sampling rate is $400 \mathrm{MHz}$, the IF is $30 \mathrm{MHz}$, the Doppler frequency shift is $300 \mathrm{~Hz}$, the initial code phase is 905 chips, the $C / N_{0}$ range is $34 \mathrm{~dB} \cdot \mathrm{Hz} \leq \mathrm{Cl}$ $N_{0} \leq 43 \mathrm{~dB} \cdot \mathrm{Hz}$ with a $1 \mathrm{~dB}$ step, the frequency search step is $300 \mathrm{~Hz}$, the frequency search range is $-1200 \mathrm{~Hz}$ $\leq f \leq 1200 \mathrm{~Hz}$, Monte Carlo simulation times are $N=1000$, and $\alpha=0.9$ in the SEAQT method. 


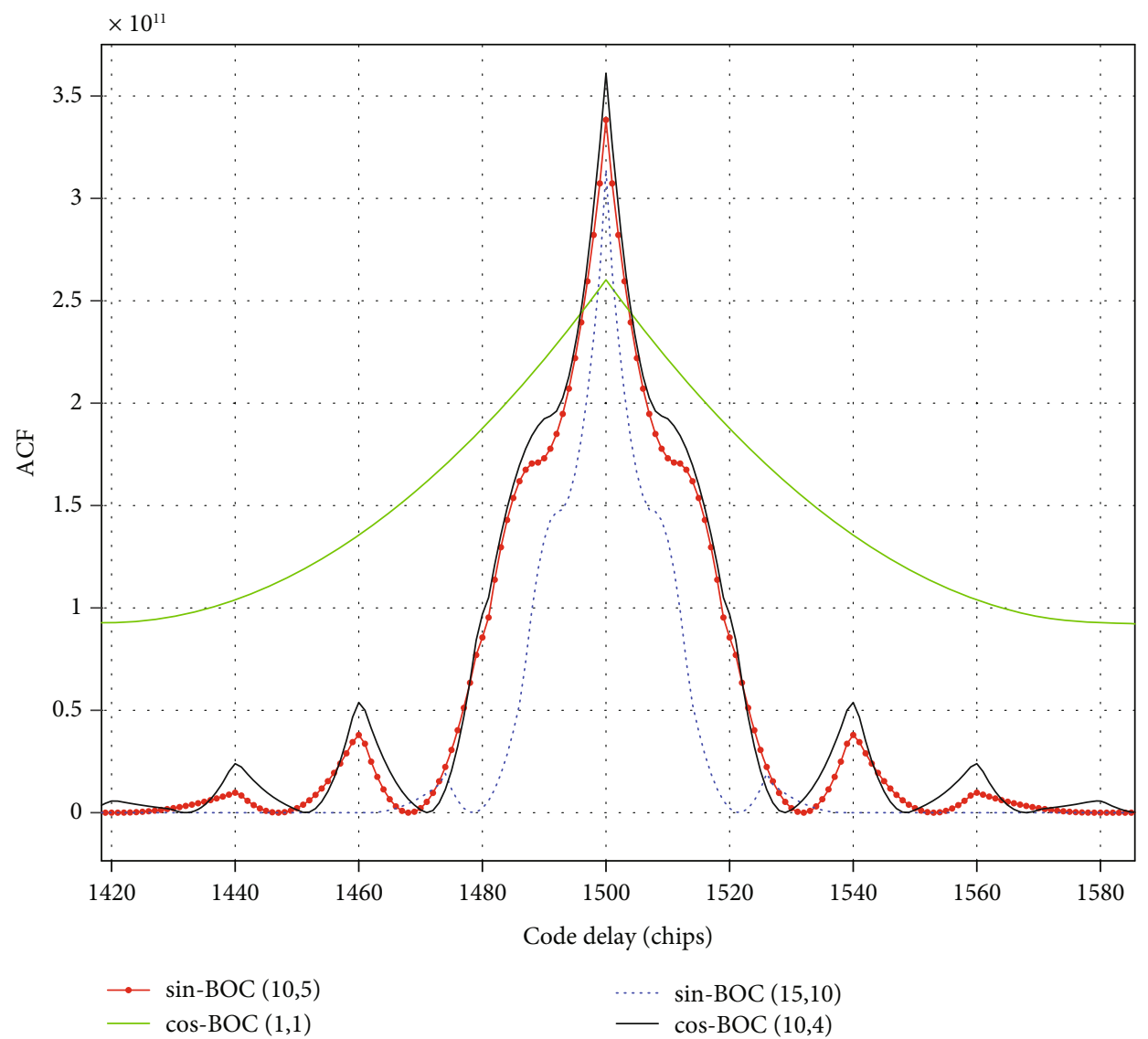

FIgUre 14: ACF of the four BOC signals.
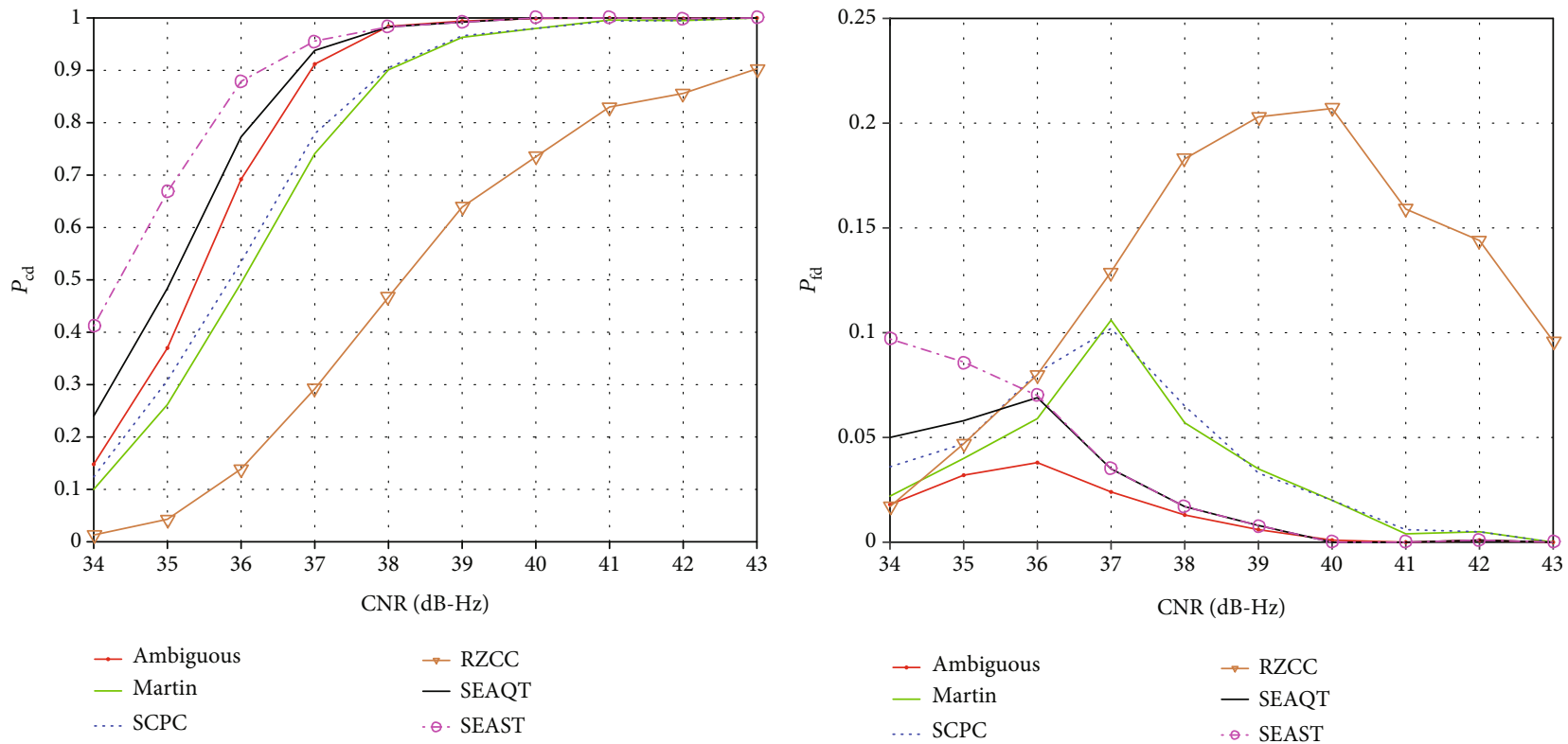

FIGURE 15: Correct detection probability and false detection probability for sin-BOC $(10,2)$.

As shown in Figure 15, the correct detection probability $P_{c d}$ of the SEAST method is the highest, followed by the SEAQT method. Taking 90\% correct detection probability as an example, the SEAQT algorithm improves by about $1.2 \mathrm{~dB}$ compared with the SCPC algorithm. As $C / N_{0} \geq 37$ $\mathrm{dB} \cdot \mathrm{Hz}$, the false detection probability $P_{\mathrm{fd}}$ of SEA is less than 

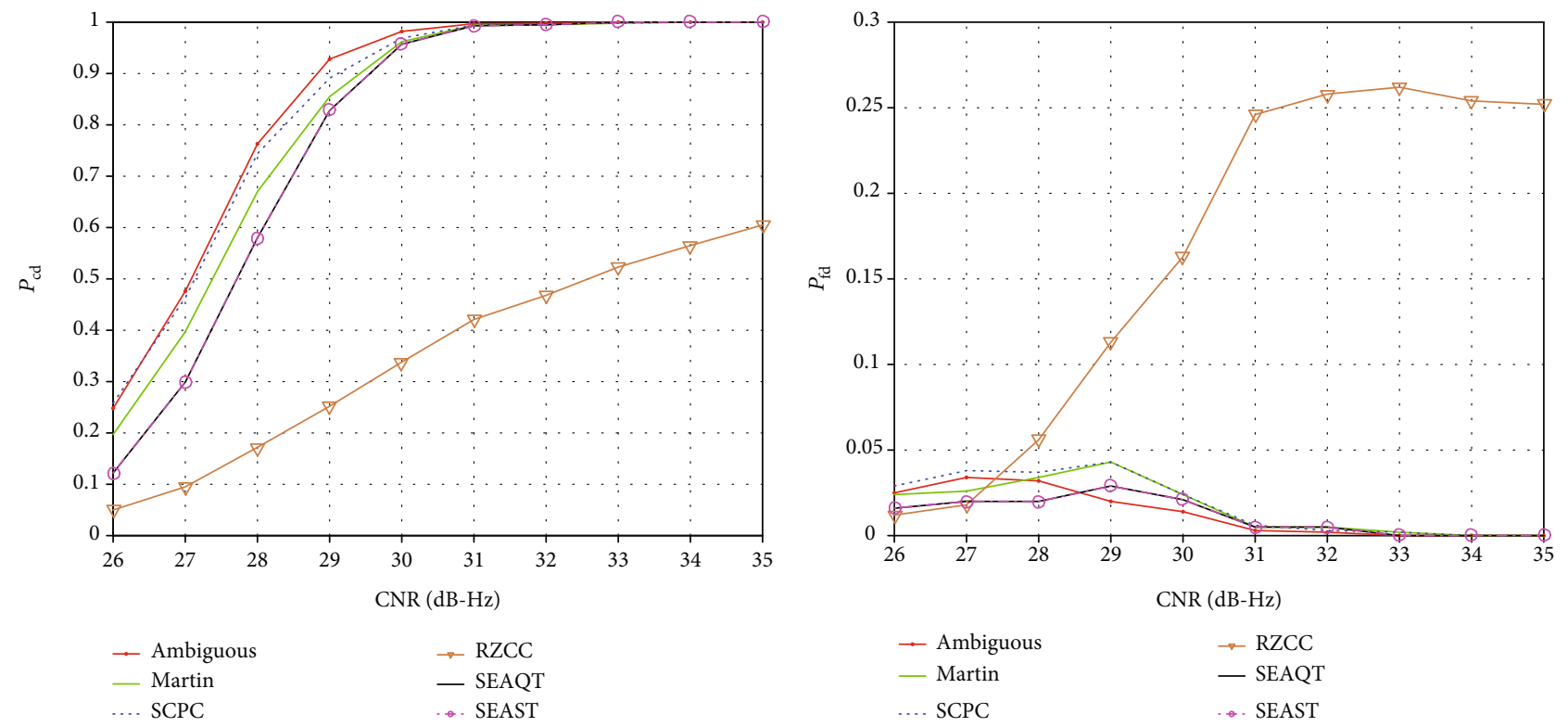

FIGURE 16: Correct detection probability and false detection probability for sin-BOC $(1,1)$.

that of the Martin method or SCPC method. For the RZCC algorithm, both $P_{\mathrm{cd}}$ and $P_{\mathrm{fd}}$ are not performing well. In some cases, $P_{\mathrm{fd}}$ of RZCC exceeds $20 \%$.

The second set of simulation conditions is set as follows: for the sin-BOC $(1,1)$ signal, the false alarm rate is $P_{\mathrm{fa}}=10^{-4}$, the sampling rate is $400 \mathrm{MHz}$, the IF is $40 \mathrm{MHz}$, the Doppler frequency shift is $580 \mathrm{~Hz}$, the initial code phase is 1500 chips, the $C / N_{0}$ range is $26 \mathrm{~dB} \cdot \mathrm{Hz} \leq C / N_{0} \leq 35 \mathrm{~dB} \cdot \mathrm{Hz}$ with a $1 \mathrm{~dB}$ step, the frequency search step is $300 \mathrm{~Hz}$, the frequency search range is $-1200 \mathrm{~Hz} \leq f \leq 1200 \mathrm{~Hz}$, Monte Carlo simulation times are $N=1000$, and $\alpha=0.7$ in the SEAQT method.

As shown in Figure 16, the correct detection probability $P_{c d}$ of SEAQT/SEAST is similar, which is slightly lower than that of the Martin method and SCPC, but the false detection probability $P_{\mathrm{fd}}$ performs better than that of the Martin method and SCPC method. The reason why SEA does not capture the BOC $(n, n)$ signal well is that the second peak of the BOC $(n, n)$ signal is only $1 / 4$ of the main peak, which leads to the main peak after enfoldment is 1.5 times than the former one. In other words, the enfoldment signal may not be high enough to exceed the acquisition threshold, which results in a low detection probability. As for the RZCC algorithm, it performs even worse than SEA. When $C / N_{0} \geq$ $31 \mathrm{~dB} \cdot \mathrm{Hz}, P_{\mathrm{fd}}$ of RZCC reaches more than $25 \%$, but $P_{\mathrm{cd}}$ is less than $60 \%$.

The third set of simulation conditions is set as follows: for the cos-BOC $(15,2.5)$ signal, the false alarm rate is $P_{\mathrm{fa}}=10^{-4}$, the sampling rate is $400 \mathrm{MHz}$, the IF is $50 \mathrm{MHz}$, the Doppler frequency shift is $-330 \mathrm{~Hz}$, the initial code phase is 3220 chips, the $C / N_{0}$ range is $36 \mathrm{~dB} \cdot \mathrm{Hz} \leq C / N_{0} \leq 45 \mathrm{~dB} \cdot \mathrm{Hz}$ with a $1 \mathrm{~dB}$ step, the frequency search step is $300 \mathrm{~Hz}$, the frequency search range is $-1200 \mathrm{~Hz} \leq f \leq 1200 \mathrm{~Hz}$, Monte Carlo simulation times are $N=1000$, and $\alpha=0.95$ in the SEAQT method.

As shown in Figure 17, the correct detection probability $P_{c d}$ of SEAST is the highest, followed by the SEAQT. Taking
90\% correct detection probability as an example, the SEAQT algorithm improves by $2.1 \mathrm{~dB}$ compared with the Martin algorithm. When $C / N_{0} \geq 38 \mathrm{~dB} \cdot \mathrm{Hz}$, the false detection probability $P_{\mathrm{fd}}$ of SEA is less than that of the Martin method or SCPC method. As for the RZCC algorithm, $P_{\mathrm{fd}}$ of RZCC is smaller than SEA under certain circumstances. However, $P_{c d}$ of RZCC performs worse than that of SEA. That is to say, RZCC sacrifices $P_{\mathrm{cd}}$ for the good performance of $P_{\mathrm{fd}}$.

The acquisition performances of the four methods are compared in Table 1 . The $C / N_{0}$ corresponding to $P_{c d}=90 \%$ and the average $P_{\mathrm{fd}}$ are compared. As can be seen from Table 1 , when the modulation order is low $(\sin -\operatorname{BOC}(1,1))$, the $C / N_{0}$ required for $P_{c d}=90 \%$ of the SEA algorithm is slightly higher than that of the Martin algorithm and SCPC algorithm, and the average $P_{\mathrm{fd}}$ is lower than that of the Martin algorithm and SCPC algorithm; when the modulation order is high, the $C / N_{0}$ required for $P_{\mathrm{cd}}=90 \%$ and the average $P_{\mathrm{fd}}$ of the SEA algorithm are lower than those of the Martin and SCPC algorithm. By comparing comprehensively, the SEA algorithm is superior to the Martin algorithm and SCPC algorithm.

4.4. $\alpha$ Value Impact Analysis. The $\alpha$ value of the SEAQT algorithm is used for the first detection, which is a screening of the capture statistic to reduce the probability of false detection. When the $\alpha$ value approaches zero, the SEAQT will degenerate into SEAST. Considering that a detection error is more likely to occur when the modulation order is high, a larger $\alpha$ value can be used to minimize $P_{\mathrm{fd}}$ in this case. As the enfoldment gain of the low-order BOC signal is low, the capture statistic is difficult to exceed the threshold, which makes the false detection possibility low, and $P_{\mathrm{fd}}$ is not prone to occur. Therefore, the value of $\alpha$ can be smaller than the high-order BOC. 

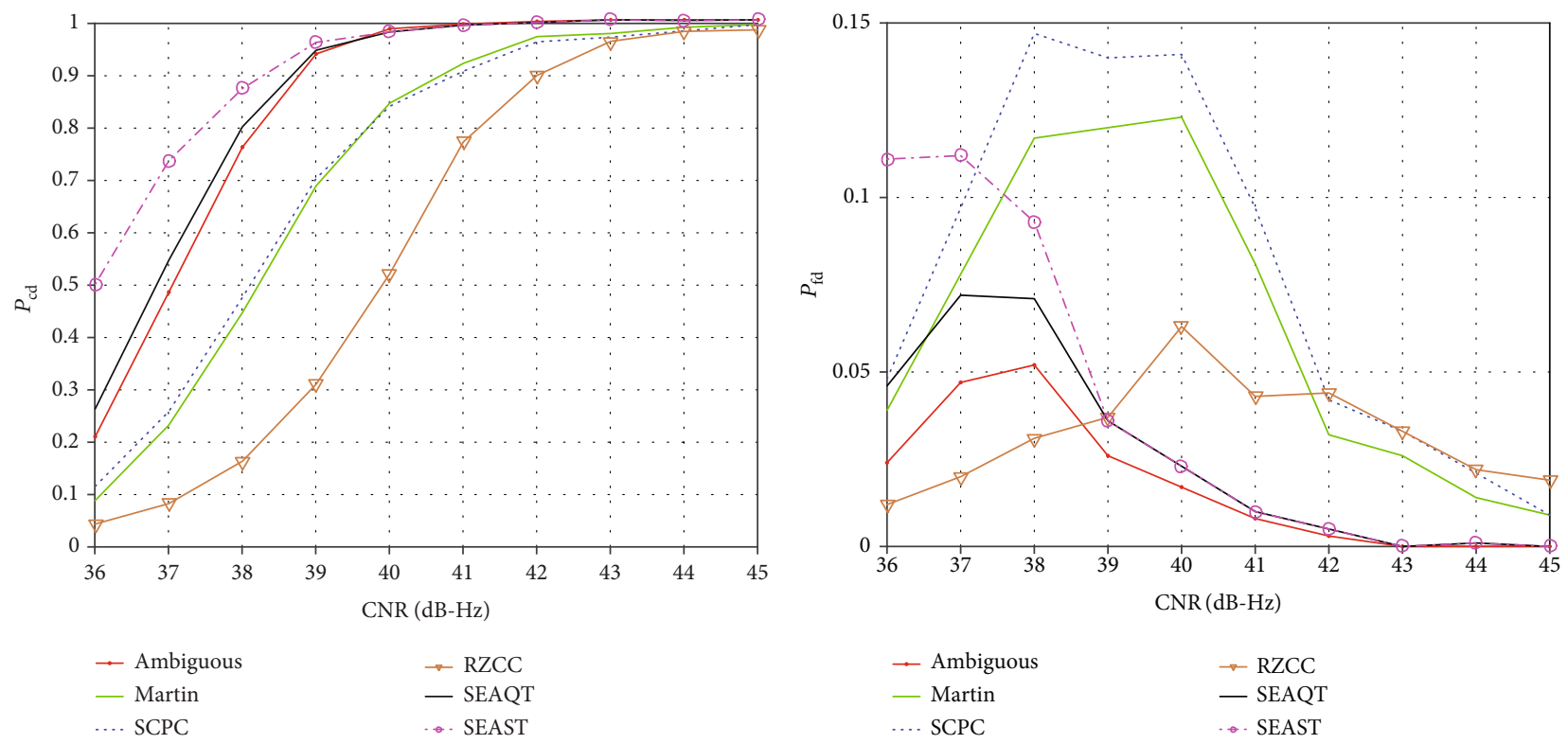

FIGURE 17: Correct detection probability and false detection probability for cos-BOC $(15,2.5)$.

TABLE 1: Comparison of capture performance for different algorithms.

\begin{tabular}{lccc}
\hline BOC signal & Algorithm & $C / N_{0}\left(P_{\mathrm{cd}}=90 \%\right)$ & Average $P_{\mathrm{fd}}$ \\
\hline & Martin & $38.0 \mathrm{~dB} \cdot \mathrm{Hz}$ & $3.48 \%$ \\
$\sin -\mathrm{BOC}(10,2)$ & SCPC & $38.0 \mathrm{~dB} \cdot \mathrm{Hz}$ & $3.95 \%$ \\
& SEAQT & $36.8 \mathrm{~dB} \cdot \mathrm{Hz}$ & $2.38 \%$ \\
& SEAST & $36.3 \mathrm{~dB} \cdot \mathrm{Hz}$ & $3.14 \%$ \\
\hline \multirow{5}{*}{$\sin -\mathrm{BOC}(1,1)$} & Martin & $29.4 \mathrm{~dB} \cdot \mathrm{Hz}$ & $1.63 \%$ \\
& SCPC & $29.1 \mathrm{~dB} \cdot \mathrm{Hz}$ & $1.82 \%$ \\
& SEAQT & $29.6 \mathrm{~dB} \cdot \mathrm{Hz}$ & $1.16 \%$ \\
& SEAST & $29.6 \mathrm{~dB} \cdot \mathrm{Hz}$ & $1.16 \%$ \\
\hline \multirow{5}{*}{$\cos -\mathrm{BOC}(15,2.5)$} & Martin & $40.8 \mathrm{~dB} \cdot \mathrm{Hz}$ & $6.39 \%$ \\
& SCPC & $41.0 \mathrm{~dB} \cdot \mathrm{Hz}$ & $7.75 \%$ \\
& SEAQT & $38.7 \mathrm{~dB} \cdot \mathrm{Hz}$ & $2.64 \%$ \\
& SEAST & $38.4 \mathrm{~dB} \cdot \mathrm{Hz}$ & $3.91 \%$ \\
\hline
\end{tabular}

Taking the sin-BOC $(10,2)$ signal as an example, when $P_{\mathrm{fa}}=10^{-4}, \mathrm{C} / \mathrm{N}_{0}=36 \mathrm{~dB} \cdot \mathrm{Hz}$, the range of $\alpha$ is between $[0,1]$ with step 0.05, and 1000 simulations are carried out. The relationship curves between $P_{\mathrm{cd}}$ and $P_{\text {fd }}$ versus $\alpha$ are shown in Figure 18. As can be seen from the figure, in order to obtain higher $P_{\mathrm{cd}}$ and lower $P_{\mathrm{fd}}, \alpha=0.65$ should be taken; in order to ensure lower $P_{\text {fd }}, \alpha=0.95$ should be taken. With the increase in the $\alpha$ value especially when $\alpha>0.7, P_{\text {cd }}$ gradually decreases while the size of the $\alpha$ value has a little effect on $P_{\mathrm{fd}}$.

4.5. Complexity Analysis of Algorithms. When the local code is correlated with the received signal in the frequency domain, the frequency band number of the captured signal is recorded as $n_{\mathrm{f}}$ and the coherence integration length is recorded as $N_{0}$. The computational complexity of a FFT operation is counted as $N_{\mathrm{FFT}}$, the computational complexity of filtering is marked as $N_{\text {filter }}$, the computational complexity of complex multiplication is marked as $N_{\mathrm{m}}$, and the computational complexity of real addition is marked as $N_{\mathrm{a}}$.

In addition to FFT operation, the Martin algorithm needs one filtering for the $I / Q$ branch separately and one complex multiplication for upper and lower sidebands. Then, the complexity of the Martin algorithm is

$$
N_{\text {Andia }}=n_{\mathrm{f}}\left(4 N_{\text {FFT }}+2 N_{\text {filter }}+2 N_{\mathrm{m}}+3 N_{\mathrm{a}}\right) \text {. }
$$

Among them, $4 N_{\mathrm{FFT}}$ is the sum of the FFT computational complexity of the upper/lower sideband $I / Q$ branch, $2 N_{\text {filter }}$ is the sum of the computational complexity of the $I / Q$ branch filter, $2 N_{\mathrm{m}}$ is the sum of the computational complexity of the upper and lower sideband complex multiplication, $3 N_{\mathrm{a}}$ is the sum of the computational complexity of the upper/lower side bands of $I^{2}$ plus $Q^{2}$ and the upper $I^{2}+Q^{2}$ plus lower $I^{2}+Q^{2}$ side band.

Similarly, the complexity of the SCPC algorithm is

$$
N_{\mathrm{SCPC}}=n_{\mathrm{f}}\left(4 N_{\mathrm{FFT}}+2 N_{\text {filter }}+3 N_{\mathrm{a}}\right) \text {. }
$$

In the SEAQT algorithm, the computational complexity of the first threshold decision is counted as $N_{\text {detection, }}$ the computational complexity of searching three peaks and two valleys is counted as $N_{\text {find }}$, and the computational complexity of enfoldment is counted as $N_{\text {fold }}$. Therefore, the complexity of the SEAQT algorithm is as follows:

$$
N_{\mathrm{SEAQT}}=n_{\mathrm{f}}\left(2 N_{\mathrm{FFT}}+N_{\mathrm{a}}+N_{\text {detection }}+N_{\text {find }}+N_{\text {fold }}\right) \text {. }
$$

The complexity of the SEAST algorithm is as follows:

$$
N_{\text {SEAST }}=n_{\mathrm{f}}\left(2 N_{\mathrm{FFT}}+N_{\mathrm{a}}+N_{\text {find }}+N_{\text {fold }}\right) \text {. }
$$



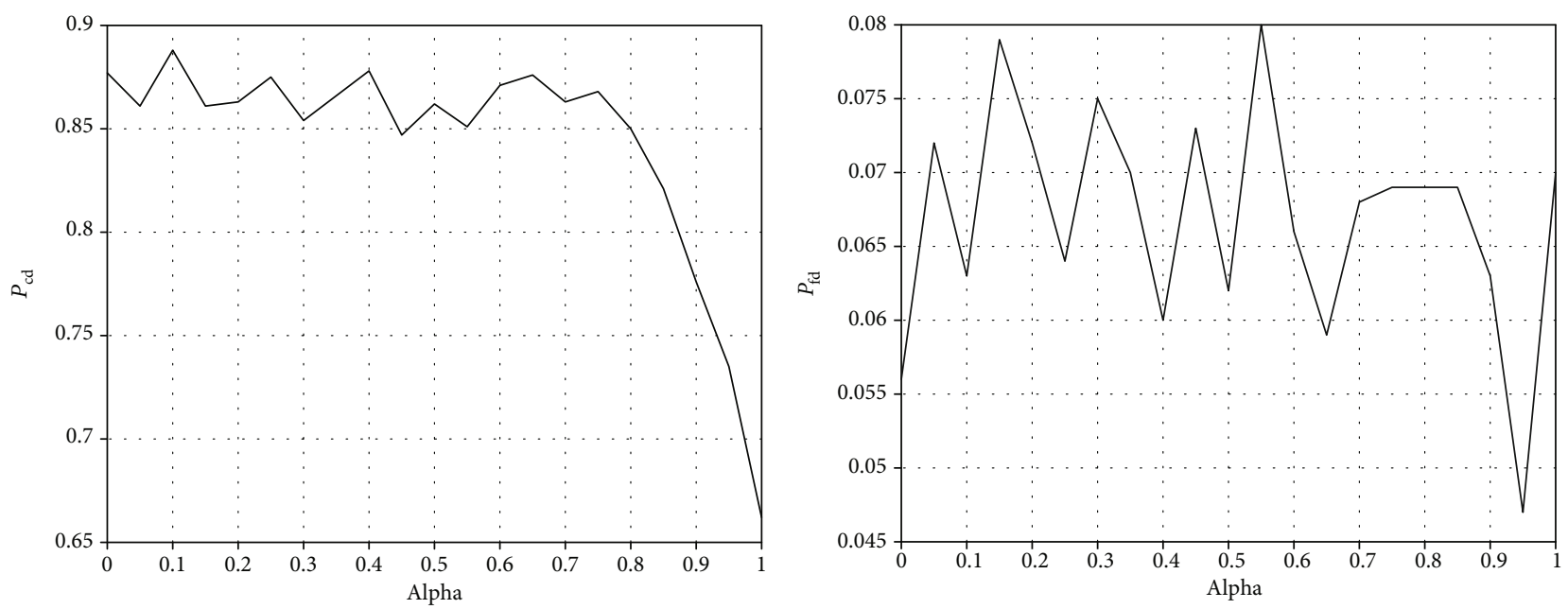

Figure 18: The relationship curves between $P_{\mathrm{cd}}$ and $P_{\mathrm{fd}}$ versus $\alpha$.

Since the SEA algorithm is not computationally intensive for searching the three peaks and two valleys and the enfoldment calculation is a simple multiplication and addition, the complexity of the SEA algorithm is lower compared with that of the Martin algorithm and SCPC algorithm.

\section{Conclusions}

Aimed at the acquisition ambiguity of $\operatorname{BOC}(m, n)$ signals, this paper proposes the SEA method, which is divided into SEAQT and SEAST methods. Both methods can solve the ambiguity of BOC signals. When $M \geq 2$, the main peak of SEA is $10 \lg \left(\left(2(M-1)^{2}+M^{2}\right) /(M-2)^{2}\right) \mathrm{dB}$ stronger than the secondary peak, which greatly improves the peak value. It turns out that the false detection probability $P_{\mathrm{fd}}$ of the SEAQT method performs better than that of SEAST through comparative analysis.

The simulation results show that the SEA can be applied to the sin-BOC signal and cos-BOC signal, with even and odd modulation order. The acquisition performances of SEA, Martin, and SCPC are analyzed and compared. Except for BOC $(n, n)$ signals, the acquisition performance of the SEAQT method is better than that of the Martin method and SCPC method in other cases.

Considering that the modulation order is high, the detection error is more likely to occur and a larger $\alpha$ value can be used to minimize $P_{\mathrm{fd}}$ in this case. When the modulation order is low, the value of $\alpha$ can be smaller. This paper takes the sin-BOC $(10,2)$ signal as an example to analyze the effect of $\alpha$ on $P_{\mathrm{cd}}$ and $P_{\mathrm{fd}}$. Various $\alpha$ values can be selected according to different needs for $P_{\mathrm{cd}}$ and $P_{\mathrm{fd}}$.

As above, both SEAQT and SEAST algorithms are superior to the RZCC algorithms. As for cos-BOC $(15,2.5)$, RZCC sacrifices $P_{\mathrm{cd}}$ for the good performance of $P_{\mathrm{fd}}$. In the other two cases, SEAQT and SEAST algorithms are much better than RZCC. Compared with the Martin algorithm and SCPC algorithm, the SEA algorithm does not need to use filters or an auxiliary signal. According to the comprehensive comparison, the SEA algorithm is better than the Martin, SCPC, and RZCC algorithms.

\section{Data Availability}

The data is simulated by MATLAB. All of the used data can be verified and replicated by reading the manuscript and simulation.

\section{Conflicts of Interest}

The authors declare that they have no conflicts of interest.

\section{References}

[1] J. W. Betz, "The offset carrier modulation for GPS modernization," in Proceedings of International National Technical Meeting, Institute of Navigation, pp. 639-648, San Diego, CA, USA, January 1999.

[2] J. W. Betz, "Binary offset carrier modulations for radionavigation," Navigation, vol. 48, no. 4, pp. 227-246, 2002.

[3] Z. Yao, M. Lu, and Z. Feng, "Unambiguous sine-phased binary offset carrier modulated signal acquisition technique," IEEE Transactions on Wireless Communications, vol. 9, no. 2, pp. 577-580, 2010.

[4] J. W. Betz, "Design and performance of code tracking for the GPS M code signal," in Proceedings of International National Technical Meeting, Institute of Navigation, pp. 2140-2150, Salt Lake City, UT, USA, January 2000.

[5] P. Fishman and J. W. Betz, "Predicting performance of direct acquisition for the M code signal," in Proceedings of International National Technical Meeting, Institute of Navigation, pp. 574-582, Anaheim, CA, USA, January, 2000.

[6] J. W. Betz and P. Capozza, "System for direct acquisition of received signals," US Patent 2004/0071200 A1, 2004.

[7] N. Martin, V. Leblond, G. Guillotel, and V. Heiries, "BOC $(\mathrm{x}, \mathrm{y})$ signal acquisition techniques and performances," in Proceedings of International National Technical Meeting, Institute of Navigation, pp. 188-198, Portland, OR, USA, September 2003.

[8] V. Heiries, D. Roviras, L. Ries, and V. Calmettes, “Analysis of non-ambiguous BOC signal acquisition performance," in Proceedings of International National Technical Meeting, Institute of Navigation, pp. 2611-2622, Long Beach, CA, USA, September 2004. 
[9] O. Julien, C. Macabiau, M. Cannon, and G. Lachapelle, "ASPeCT: unambiguous sine-BOC (n, n) acquisition/tracking technique for navigation applications," IEEE Transactions on Aerospace and Electronic Systems, vol. 43, no. 1, pp. 150-162, 2007.

[10] Y. Zhou, X. Hu, T. Ke, and Z. Tang, “Ambiguity mitigating technique for cosine-phased binary offset carrier signal," IEEE Transactions on Wireless Communications, vol. 11, no. 6, pp. 1981-1984, 2012.

[11] P. W. Ward, "A design technique to remove the correlation ambiguity in binary offset carrier (BOC) spread spectrum signals," in Proceedings of Annual Meeting of the Institute of Navigation \& CIGTF Guidance Test Symposium, Institute of Navigation, pp. 886-896, Albuquerque, NM, USA, June 2003.

[12] Z. Yang, Z. Huang, and S. Geng, "Acquisition performance analysis of BOC signal considering the code search step size," Journal of Computers, vol. 6, no. 7, pp. 1386-1393, 2011.

[13] F. Shen, G. Xu, J. W. Cheong, and H. Feng, "Unambiguous acquisition and tracking technique for general BOC signals," Radio Engineering, vol. 24, no. 3, pp. 840-849, 2015.

[14] H. Li and C. Yang, "An unambiguous receiving technique for $\mathrm{BOC}(\mathrm{n}, \mathrm{n})$ signal," in 2014 IEEE International Conference on Signal Processing, Communications and Computing (ICSPCC), pp. 379-383, Guilin, China, August 2014.

[15] P. Li, F. Gao, and H. Han, "A novel unambiguous acquisition scheme for Galileo modulated BOC (n, n) signals," in 11th International Conference on Wireless Communications, Networking and Mobile Computing (WiCOM 2015), Shanghai, China, September 2016.

[16] H. Xiong, S. Wang, S. Gong, M. Peng, J. Shi, and J. Tang, "Improved synchronisation algorithm based on reconstructed correlation function for BOC modulation in satellite navigation and positioning system," IET Communications, vol. 12, no. 6, pp. 743-750, 2018.

[17] F. Benedetto, G. Giunta, E. S. Lohan, and M. Renfors, "A fast unambiguous acquisition algorithm for BOC-modulated signals," IEEE Transactions on Vehicular Technology, vol. 62, no. 3, pp. 1350-1355, 2013.

[18] D.-S. Shim and J.-S. Jeon, "An unambiguous delay-andmultiply acquisition scheme for GPS L1C signals," Sensors, vol. 18 , no. 6 , article 1739, 2018.

[19] B. Kim and S. H. Kong, "Two-dimensional compressed correlator for fast acquisition of $\operatorname{BOC}(m, n)$ signals," IEEE Transactions on Vehicular Technology, vol. 63, no. 6, pp. 2662-2672, 2014.

[20] L. Yang, C. Pan, Y. Bo, and Y. Feng, "A new method for acquisition of BOC modulation signal based on refactoring theory to cross-correlation," Journal of Astronautics, vol. 30, no. 4, pp. 1675-1679, 2009.

[21] L. Yang, Research on Key Techniques of Synchronization of Navigation Signals Based on BOC Modulation, Nanjing University of Science \& Technology, 2009.

[22] Z. Deng, E. Hu, L. Yin, W. Liu, L. Yang, and Q. A. Arain, “An unambiguous tracking technique for sine-BOC $(\mathrm{kn}, \mathrm{n})$ modulated GNSS signals," Wireless Personal Communications, vol. 103, no. 2, pp. 1101-1112, 2018.

[23] Y. Lee, D. Chong, I. Song, S. Y. Kim, G. I. Jee, and S. Yoon, "Cancellation of correlation side-peaks for unambiguous BOC signal tracking," IEEE Communications Letters, vol. 16, no. 5, pp. 569-572, 2012.
[24] L. J. Garin, “The "shaping correlator", novel multipath mitigation technique applicable to Galileo $\operatorname{BOC}(1,1)$ modulation waveforms in high volume markets," in Proceedings of European Navigation Conference (ENC-GNSS) 2005, Munich, Germany, July 2005.

[25] J. Wu and A. G. Dempster, “BOC-Gated-PRN' a multipath mitigation technique for BOC(n,n) waveforms," IEEE Transactions on Aerospace and Electronic Systems, vol. 47, no. 2, pp. 1136-1153, 2011.

[26] H. Zhao, S. Du, and C. Sun, "An improved CCRW algorithm for BOC signals with odd modulation coefficient," China Satellite Navigation Conference (CSNC) 2018 Proceedings. CSNC 2018. Lecture Notes in Electrical Engineering, vol 498Springer, Singapore.

[27] C. Sun, H. Zhao, W. Feng, and S. Du, "A frequency-domain multipath parameter estimation and mitigation method for BOC-modulated GNSS signals," Sensors, vol. 18, no. 3, p. $721,2018$. 